\title{
A Lévy HJM Multiple-Curve Model with Application to CVA Computation
}

\author{
Crépey, Stéphane; Grbac, Zorana; Ngor, Nathalie; Skovmand, David
}

\author{
Document Version \\ Accepted author manuscript \\ Published in: \\ Quantitative Finance \\ DOI: \\ $10.1080 / 14697688.2014 .942232$ \\ Publication date: \\ 2015 \\ License \\ Unspecified
}

Citation for published version (APA):

Crépey, S., Grbac, Z., Ngor, N., \& Skovmand, D. (2015). A Lévy HJM Multiple-Curve Model with Application to CVA Computation. Quantitative Finance, 15(3), 401-419. https://doi.org/10.1080/14697688.2014.942232

Link to publication in CBS Research Portal

\section{General rights}

Copyright and moral rights for the publications made accessible in the public portal are retained by the authors and/or other copyright owners and it is a condition of accessing publications that users recognise and abide by the legal requirements associated with these rights.

\section{Take down policy}

If you believe that this document breaches copyright please contact us (research.lib@cbs.dk) providing details, and we will remove access to the work immediately and investigate your claim.

Download date: 26. Apr. 2023

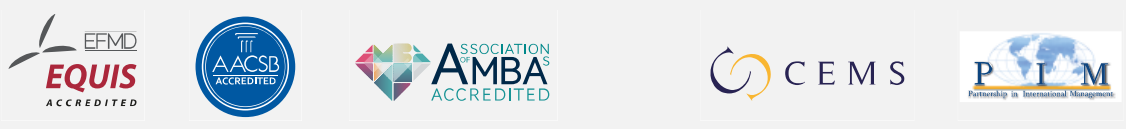




\title{
A Lévy HJM Multiple-Curve Model with Application to CVA Computation
}

\author{
Stéphane Crépey, Zorana Grbac, Nathalie Ngor, and David Skovmand \\ Journal article (Post print version)
}

This is an Accepted Manuscript of an article published by Taylor \& Francis in Quantitative

Finance on 13 August 2014, available online:

http://www.tandfonline.com/10.1080/14697688.2014.942232.

Uploaded to Research@CBS: December 2015

Available at Research@CBS

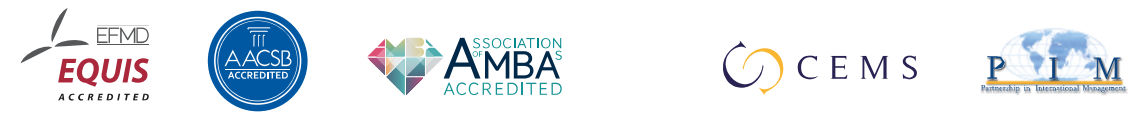




\title{
A Lévy HJM multiple-curve model with application to CVA computation
}

\author{
Stéphane Crépey ${ }^{1, *}$, Zorana Grbac ${ }^{2, \dagger}$, \\ Nathalie Ngor ${ }^{1}$, David Skovmand ${ }^{3}$ \\ ${ }^{1}$ Laboratoire de Mathématiques et Modélisation d'Évry \\ Université d'Évry Val d'Essonne, 91037 Évry Cedex, France \\ stephane.crepey@univ-evry.fr \\ nathalie.ngor@gmail.com \\ 2 Laboratoire de Probabilités et Modèles Aléatoires \\ Université Paris Diderot - Paris 7, France \\ grbac@math.univ-paris-diderot.fr \\ 3 Department of Finance \\ Copenhagen Business School, Solbjerg Plads 3, 2000 Frederiksberg, Denmark \\ dgs.fi@cbs.dk
}

July 22, 2014

\begin{abstract}
We consider the problem of valuation of interest rate derivatives in the post-crisis setup. We develop a multiple-curve model, set in the HJM framework and driven by a Lévy process. We proceed with joint calibration to OTM swaptions and co-terminal ATM swaptions of different tenors, the calibration to OTM swaptions guaranteeing that the model correctly captures volatility smile effects and the calibration to co-terminal ATM swaptions ensuring an appropriate term structure of the volatility in the model. To account for counterparty risk and funding issues, we use the calibrated multiplecurve model as an underlying model for CVA computation. We follow a reduced-form methodology through which the problem of pricing the counterparty risk and funding costs can be reduced to a pre-default Markovian BSDE, or an equivalent semi-linear PDE. As an illustration we study the case of a basis swap and a related swaption, for which we compute the counterparty risk and funding adjustments.
\end{abstract}

Keywords: interest rate derivative, multiple-curve term structure model, Lévy process, credit valuation adjustment (CVA), funding.

\section{Introduction}

As a consequence of the crisis various new phenomena appeared in the fixed income markets. A variety of spreads have developed, notably Libor-OIS swap spreads and basis swap spreads, an issue known as multiple-curves (see, among others, Kijima, Tanaka, and Wong (2009), Kenyon (2010), Henrard (2007, 2010), Bianchetti (2010), Mercurio (2010b, 2010a), Fujii, Shimada, and Takahashi (2011, 2010), Moreni and Pallavicini (2014) and Bianchetti

*The research of S. Crépey benefited from the support of the "Chair Markets in Transition" under the aegis of Louis Bachelier laboratory, a joint initiative of École Polytechnique, Université d'Évry Val d'Essonne and Fédération Bancaire Française

'Z. Grbac acknowledges the financial support from the DFG Research Center MATHEON, Project E13. 
and Morini (2013)). In addition, counterparty risk and funding costs have become major issues in OTC derivative transactions. To account for these, credit/debt valuation adjustments CVA/DVA and different kinds of funding valuation adjustment have been introduced (see Brigo, Morini, and Pallavicini (2013) and Crépey, Bielecki, and Brigo (2014), respectively in a more financial and mathematical perspective, for recent references in book form). We refer to an aggregate adjustment as a TVA, which stands for total valuation adjustment, reserving the term CVA for a strict credit valuation adjustment. In the literature, the acronym CVA is often used also in a broader sense, which then corresponds to our TVA (this is the reason why the name CVA was also preferred in the title and the abstract of the paper, however in the rest of the paper we consistently use the terms TVA and CVA in the sense explained above).

From a risk-management perspective, this new paradigm has created a need for multiplecurve interest rate models allowing for practical TVA computations, for which, in particular, a short rate process $r_{t}$ and a parsimonious Markov structure are required. A few suitable models which come to mind are the HJM multiple-curve model of Fujii and Takahashi (2011) and the "parsimonious" (in reference to the above Markov requirement) models of Moreni and Pallavicini $(2014,2013)$. Moreni and Pallavicini (2014) are the first to apply the HJM reconstruction formula, normally used to compute zero coupon bond prices, to define the FRA rates. Our model construction in this paper is similar in spirit. However, none of the mentioned papers studies the application to TVA computation. Moreover, a powerful modeling ingredient is the use of Lévy drivers, as opposed to Brownian drivers in the three mentioned papers. Specifically, we devise a HJM multiple-curve model driven by a two-dimensional Lévy process, with a built-in HJM fit to the initial Libor and OIS term structures and with a two-dimensional Markov structure. Lévy drivers were already used with the same motivation in Crépey, Grbac, and Nguyen (2012), but the Libor rates were defined in terms of the so-called Libor bonds reflecting the credit and liquidity risk of the Libor contributing banks. The main motivation was to explain in an economically satisfying way the spreads between the Libor and the OIS rates. Since Libor bonds are not traded assets, we choose in this paper to follow Mercurio (2010a) in modeling the Libor FRA rates (see the defining equation (9) below). The Libor FRA rates are directly observable up to the maturity of one year and for longer maturities they can be bootstrapped from Libor swap rates. The model of Mercurio (2010a) is developed in a standard Libor market model setup, which is less suitable for TVA computations, as it does not allow for low-dimensional Markovian representations. By contrast, in the HJM framework of this paper, we can access the short rate process $r_{t}$, which is needed for discounting in TVA computations.

The paper is organized as follows. In Sect. 2 we present the Lévy HJM multiple-curve model. Pricing formulas for the most common interest rate derivatives such as swaps and basis swaps, caplets and swaptions are provided in Sect. 3. Sect. 4 deals with the calibration of the model to OTM swaptions guaranteeing that the model correctly captures volatility smile effects (in strike) and to co-terminal ATM swaptions ensuring an appropriate term structure of the volatility in the model. This is important in view of the targeted application to TVA computations on multiple-curve products, which is the topic of Sect. 5, where we use the calibrated multiple-curve model as an underlying model for TVA computation on a basis swap and a related swaption. 


\section{Multiple-Curve Model}

In this section we develop a Lévy driven HJM multiple-curve model for the Libor FRA rates, which are the underlying rates for most interest rate derivatives, and the OIS bond prices implied by the overnight indexed swaps (OIS), which are used for discounting.

\subsection{Driving Process}

Let a filtered probability space $\left(\Omega, \mathcal{F}_{\bar{T}}, \mathbb{F}, \mathbb{P}\right)$, where $\bar{T}$ is a finite time horizon and $\mathbb{P}$ is a risk-neutral pricing measure, be fixed. The filtration $\mathbb{F}=\left(\mathcal{F}_{t}\right)_{t \in[0, \bar{T}]}$ satisfies the usual conditions. The driving process $Y=\left(Y_{t}\right)_{0 \leq t \leq \bar{T}}$ is assumed to be an $\mathbb{F}$ adapted, $\mathbb{R}^{n}$ valued Lévy process (see Cont and Tankov (2003) and Sato (1999)). The characteristic function of $Y_{t}$ is given by the Lévy-Khintchine formula, in which $u$ denotes a row-vector in $\mathbb{R}^{n}$ :

$$
\mathbb{E}\left[e^{\mathrm{i} u Y_{t}}\right]=\exp \left(t\left(\mathrm{i} u b-\frac{1}{2} u c u^{\top}+\int_{\mathbb{R}^{n}}\left(e^{\mathrm{i} u x}-1-\mathrm{i} u h(x)\right) F(d x)\right)\right),
$$

where $b \in \mathbb{R}^{n}, c$ is a symmetric, nonnegative definite real-valued $n$ dimensional matrix and $F$ is a Lévy measure on $\mathbb{R}^{n}$, i.e. $F(\{0\})=0$ and $\int_{\mathbb{R}^{n}}\left(|x|^{2} \wedge 1\right) F(d x)<\infty$. The function $h: \mathbb{R}^{n} \rightarrow \mathbb{R}^{n}$ is a suitable truncation function. We assume that there exist constants $\mathcal{K}_{1}, \mathcal{K}_{2}, \varepsilon>0$ such that

$$
\int_{|x|>1} \exp (u x) F(d x)<\infty, u \in\left[-(1+\varepsilon) \mathcal{K}_{1},(1+\varepsilon) \mathcal{K}_{2}\right]^{n} .
$$

As is well-known, condition (2) holds if and only if $\mathbb{E}\left[\exp \left(u Y_{t}\right)\right]<\infty$ for all $0 \leq t \leq \bar{T}$ and $u \in\left[-(1+\varepsilon) \mathcal{K}_{1},(1+\varepsilon) \mathcal{K}_{2}\right]^{n}$ (cf. Theorem 25.3 in Sato (1999)). Moreover, (2) ensures that $h(x)=x$ can be chosen as truncation function. Hence, $Y$ is a special semimartingale, with the canonical representation

$$
Y_{t}=b t+\sqrt{c} W_{t}+\int_{0}^{t} \int_{\mathbb{R}^{n}} x(\mu-\nu)(d s, d x), \quad t \in[0, \bar{T}],
$$

where $\mu$ is the random measure of the jumps of $Y, \nu$ is the $\mathbb{P}$ compensator of $\mu$ given by $\nu(d s, d x)=F(d x) d s, \sqrt{c}$ is a measurable version of a square-root of the matrix $c$ and $W$ is a $\mathbb{P}$ standard Brownian motion. The cumulant generating function associated with the Lévy process $Y$ is denoted by $\psi$. For any row-vector $z \in \mathbb{C}^{n}$ such that $\Re z \in\left[-(1+\varepsilon) \mathcal{K}_{1},(1+\right.$ $\left.\varepsilon) \mathcal{K}_{2}\right]^{n}$, we have

$$
\psi(z)=z b+\frac{1}{2} z c z^{\top}+\int_{\mathbb{R}^{n}}\left(e^{z x}-1-z x\right) F(d x) .
$$

Consequently, (1) can be written as:

$$
\mathbb{E}\left[e^{\mathrm{i} u Y_{t}}\right]=\exp (t \psi(\mathrm{i} u)) .
$$

\subsection{Multiple-Curve Dynamics}

In this section we present the multiple-curve model for the OIS bond prices and the Libor FRA rates. The dynamics of the OIS bond prices $\left(B_{t}(T)\right)_{0 \leq t \leq T \leq \bar{T}}$ are modeled in a HJM fashion as follows (see e.g. Eberlein and Raible (1999)):

$$
B_{t}(T)=\frac{B_{0}(T)}{B_{0}(t)} \exp \left(\int_{0}^{t}(A(s, t)-A(s, T)) d s+\int_{0}^{t}(\Sigma(s, t)-\Sigma(s, T)) d Y_{s}\right),
$$


where $A(s, T)$ and $\Sigma(s, T)$ are deterministic, real-valued, respectively $\mathbb{R}^{n}$ valued, functions defined on the set $\{(s, T) \in[0, \bar{T}] \times[0, \bar{T}]: 0 \leq s \leq T\}$, with paths continuously differentiable in the second variable. We assume a bounded, positive initial term structure $\left(B_{0}(T)\right)_{0 \leq T \leq \bar{T}}$ and volatility structure $\Sigma(s, T)=\left(\Sigma^{i}(s, T)\right)_{i=1, \ldots, n}$ such that $0 \leq \Sigma^{i}(s, T) \leq \frac{\mathcal{K}}{2}$ for every $0 \leq s \leq T \leq \bar{T}$ and $i \in\{1,2, \ldots, n\}$, where $\mathcal{K}:=\min \left\{\mathcal{K}_{1}, \mathcal{K}_{2}\right\}$ for the constants $\mathcal{K}_{1}, \mathcal{K}_{2}$ from (2). Moreover, $A(s, T)=\psi(-\Sigma(s, T))$, for every $0 \leq s \leq T$, which is a classical Lévy HJM drift condition guaranteeing absence of arbitrage between OIS bonds. The OIS discount factor process $\beta=\left(\beta_{t}\right)_{0 \leq t \leq \bar{T}}$ defined by $\beta_{t}=\exp \left(-\int_{0}^{t} r_{s} d s\right)$, where $r$ represents the short rate process, can be written as

$$
\beta_{t}=B_{0}(t) \exp \left(-\int_{0}^{t} A(s, t) d s-\int_{0}^{t} \Sigma(s, t) d Y_{s}\right),
$$

so that

$$
B_{t}(T)=B_{0}(T) \exp \left(\int_{0}^{t}\left(r_{s}-A(s, T)\right) d s-\int_{0}^{t} \Sigma(s, T) d Y_{s}\right) .
$$

Now we define the Libor FRA rates and specify their dynamics. More precisely, we introduce the following quantities:

$$
F_{t}(T, S)=(S-T) \mathbb{E}_{t}^{S}\left[L_{T}(T, S)\right]
$$

where $0 \leq t \leq T \leq S$. Here $L_{T}(T, S)$ denotes a $T$ spot Libor rate fixed at time $T$ for the time interval $[T, S]$ and $\mathbb{E}_{t}^{S}$ denotes the $\mathcal{F}_{t}$ conditional expectation with respect to the $S$ forward martingale measure $\mathbb{P}^{S}$ (see $(10)$ ), where $\mathbb{F}=\left(\mathcal{F}_{t}\right)_{0 \in[0, \bar{T}]}$ is the reference filtration. These quantities are exactly the $(S-T) \times$ Libor FRA rates, as defined in Mercurio (2010a). Defined using $\mathbb{E}_{t}^{S}\left[L_{T}(T, S)\right]$ in $(9)$, FRA rates are by their very definition martingales under $S$ forward martingale measures, consistent with model-free arbitrage requirements. Modeling $(S-T) \times$ FRA rates instead of the FRA rates themselves ("interest charge instead of interest rate") gives rise to slightly simpler formulas in our setup. By a slight abuse of terminology, we will refer also to $F_{t}(T, S)$ as the FRA rate. The definition of $F_{t}(T, S)$ implies the two following modeling requirements:

(i) $F_{t}(T, S) \geq 0$, for every $t$

(ii) $F .(T, S)$ is a $\mathbb{P}^{S}$ martingale, where the $\mathbb{P}^{S}$ forward martingale measure is characterized in terms of $\mathbb{P}$ by

$$
\left.\frac{d \mathbb{P}^{S}}{d \mathbb{P}}\right|_{\mathcal{F}_{t}}=\frac{\beta_{t} B_{t}(S)}{B_{0}(S)}, 0 \leq t \leq S
$$

Both requirements are direct consequences of (9) if the Libor rate $L_{T}(T, S)$ is nonnegative, which is implied by market observations. We model $F_{t}(T, S)$, for all $0 \leq t \leq T \leq S$, as

$$
F_{t}(T, S)=F_{0}(T, S) \exp \left(\int_{0}^{t} \alpha(s, T, S) d s+\int_{0}^{t} \varsigma(s, T, S) d Y_{s}\right)
$$

where $\alpha(s, T, S)$ is a drift term and $\varsigma(s, T, S)$ a volatility structure. We assume that $\alpha(s, T, S)$ and $\varsigma(s, T, S)$ are deterministic, real-valued, respectively $\mathbb{R}^{n}$ valued, functions defined on the set $\{(s, T, S) \in[0, \bar{T}] \times[0, \bar{T}] \times[0, \bar{T}]: 0 \leq s \leq T \leq S\}$ and such that the above integrals are well-defined. We assume a bounded, positive initial term structure $\left(F_{0}(T, S)\right)_{0 \leq T \leq S \leq \bar{T}}$ and volatility structure $\varsigma(s, T, S)=\left(\varsigma^{i}(s, T, S)\right)_{i=1, \ldots, n}$ such that 
$0 \leq \varsigma^{i}(s, T, S) \leq \frac{\mathcal{K}}{2}$, for every $0 \leq s \leq T \leq S$ and $i \in\{1, \ldots, n\}$. With this specification, requirement (i) is satisfied automatically and (ii) holds if the following drift condition is satisfied

$$
\begin{aligned}
\alpha(s, T, S)= & -\varsigma(s, T, S) b_{s}^{S}-\frac{1}{2} \varsigma(s, T, S) c_{s} \varsigma(s, T, S)^{\top} \\
& \quad-\int_{\mathbb{R}^{d}}\left(e^{\varsigma(s, T, S) x}-1-\varsigma(s, T, S) x\right) F_{s}^{S}(d x) \\
= & -\psi_{s}^{S}(\varsigma(s, T, S))=\psi(-\Sigma(s, S))-\psi(\varsigma(s, T, S)-\Sigma(s, S)),
\end{aligned}
$$

where $\left(b_{s}^{S}, c_{s}, F_{s}^{S}\right)$ is the time-dependent Lévy triplet and $\psi_{s}^{S}$ the corresponding cumulant generating function of $Y$ under the forward measure $\mathbb{P}^{S}$. This result is well-known for Lévy driven term structure models (see for example Eberlein and Özkan (2005)). The last equality results from the following connection between the cumulant generating functions $\psi^{S}$ and $\psi$ of $Y$ under the measures $\mathbb{P}^{S}$ and $\mathbb{P}$ :

$$
\psi_{s}^{S}(z)=\psi(z-\Sigma(s, S))-\psi(-\Sigma(s, S))
$$

for any $z \in \mathbb{R}^{n}$ such that the above expressions are well-defined, which in turn follows by definition of the cumulant generating function and equations (8) and (10).

Remark 2.1 Note that the following generalization of (11) can also be considered, which produces an equally tractable model:

$$
F_{t}(T, S)+\Delta(T, S)=\left(F_{0}(T, S)+\Delta(T, S)\right) \exp \left(\int_{0}^{t} \alpha(s, T, S) d s+\int_{0}^{t} \varsigma(s, T, S) d Y_{s}\right),
$$

where $\Delta(T, S) \in \mathbb{R}$, for $0 \leq T \leq S$, are constant shifts. This model is known as a shifted model. The use of shifts allows one to recover a single-curve model as a special case of the multiple-curve model by setting $\Delta(T, S)=1$, for all $0 \leq T \leq S$, and $\varsigma(s, T, S)=$ $\Sigma(s, S)-\Sigma(s, T)$ in the above specification. Then $F_{t}(T, S)=\frac{B_{t}(T)}{B_{t}(S)}-1$, which is the classical relation from the single-curve model. Note, however, that in the shifted model the FRA rates can become negative with positive probability. More generally, the shifts can also be used to increase the flexibility of the model, which we found unnecessary in our case where sufficient flexibility is already ensured by maturity-dependent volatility specification for the FRA rate (cf. Sections 3.4 and 4). For different kinds of shifts used in the multiple-curve term structure literature see Mercurio (2010a) or Moreni and Pallavicini (2014).

\section{Pricing of Interest Rate Derivatives}

In this section we give an overview of the most common interest rate derivatives and provide pricing formulas in the Lévy multiple-curve model. Note that these are "clean prices", ignoring counterparty risk and assuming that funding is ensured at the OIS rate. Counterparty risk and funding valuation adjustments are computed separately, as will be explained in Sect. 5 .

\subsection{Interest Rate Derivatives With Linear Payoffs}

For interest rate derivatives with linear payoffs, the prices can be easily expressed in terms of $F_{t}(T, S)$, as we show below. 
A fixed-for-floating interest rate swap is a financial contract between two parties to exchange a stream of fixed interest payments for a stream of floating payments linked to the Libor rates, based on a specified notional amount $N$. We assume, as standard, that the Libor rate is set in advance and the payments are made in arrears. The swap is initiated at time $T_{0} \geq 0$. Denote by $T_{1}<\cdots<T_{n}$, where $T_{1}>T_{0}$, a collection of the payment dates and by $K$ the fixed rate. Then the time- $t$ value of the swap, where $t \leq T_{0}$, for the receiver of the floating rate is given by:

$$
\begin{aligned}
P_{t}^{s w} & =N \sum_{k=1}^{n} \delta_{k-1} B_{t}\left(T_{k}\right) \mathbb{E}_{t}^{T_{k}}\left[L_{T_{k-1}}\left(T_{k-1}, T_{k}\right)-K\right] \\
& =N \sum_{k=1}^{n} B_{t}\left(T_{k}\right)\left(F_{t}\left(T_{k-1}, T_{k}\right)-\delta_{k-1} K\right)
\end{aligned}
$$

where $\mathbb{E}^{T_{k}}$ is the expectation with respect to the forward measure $\mathbb{P}^{T_{k}}$ and $\delta_{k-1}=T_{k}-T_{k-1}$. The swap rate $K_{t}^{s w}$ is given by

$$
K_{t}^{s w}=\frac{\sum_{k=1}^{n} B_{t}\left(T_{k}\right) F_{t}\left(T_{k-1}, T_{k}\right)}{\sum_{k=1}^{n} \delta_{k-1} B_{t}\left(T_{k}\right)} .
$$

Remark 3.1 The Libor-OIS swap spread mentioned in the introduction is by definition the difference between the swap rate (15) of the Libor-indexed interest rate swap and the OIS rate, where the latter is given by the classical formula:

$$
K_{t}^{\text {ois }}=\frac{B_{t}\left(T_{0}\right)-B_{t}\left(T_{n}\right)}{\sum_{k=1}^{n} \delta_{k-1} B_{t}\left(T_{k}\right)} .
$$

See Filipović and Trolle (2013) for details. Thus, the Libor-OIS swap spread is given, for $0 \leq t \leq T_{0}$, by

$$
K_{t}^{s w}-K_{t}^{o i s}=\frac{\sum_{k=1}^{n} B_{t}\left(T_{k}\right) F_{t}\left(T_{k-1}, T_{k}\right)-B_{t}\left(T_{0}\right)+B_{t}\left(T_{n}\right)}{\sum_{k=1}^{n} \delta_{k-1} B_{t}\left(T_{k}\right)} .
$$

A basis swap is an interest rate swap where two streams of floating payments linked to the Libor rates of different tenors are exchanged. Both rates are set in advance and paid in arrears. We consider a basis swap with two tenor structures denoted by $\mathcal{T}^{1}=\left\{T_{0}^{1}<\right.$ $\left.\ldots<T_{n_{1}}^{1}\right\}$ and $\mathcal{T}^{2}=\left\{T_{0}^{2}<\ldots<T_{n_{2}}^{2}\right\}$, where $T_{0}^{1}=T_{0}^{2} \geq 0, T_{n_{1}}^{1}=T_{n_{2}}^{2}$, and $\mathcal{T}^{1} \subset \mathcal{T}^{2}$. The notional amount is denoted by $N$ and the swap is initiated at time $T_{0}^{1}$, where the first payments are due at $T_{1}^{1}$ and $T_{1}^{2}$. Basis swaps appeared in the markets due to the multi-curve discrepancy. A basis swap would have zero value at all times in a single-curve setup (see Section 4.4 in Crépey et al. (2012)). However, in a multiple-curve setup, the value of the basis swap is not zero and markets actually quote positive basis swap spreads, which, when added to the smaller tenor leg, give zero value to the product. More precisely, on the smaller tenor leg the floating interest rate $L_{T_{j-1}^{2}}\left(T_{j-1}^{2}, T_{j}^{2}\right)$ at $T_{j}^{2}$ is replaced by $L_{T_{j-1}^{2}}\left(T_{j-1}^{2}, T_{j}^{2}\right)+K$, for every $j=1, \ldots, n_{2}$, where $K$ is the basis swap spread. The time- $t$ value of the basis swap with spread $K$, for $t \leq T_{0}^{1}$, is given by:

$$
\begin{aligned}
P_{t}^{b s w}= & N\left(\sum_{i=1}^{n_{1}} \delta_{i-1}^{1} B_{t}\left(T_{i}^{1}\right) \mathbb{E}_{t}^{T_{i}^{1}}\left[L_{T_{i-1}^{1}}\left(T_{i-1}^{1}, T_{i}^{1}\right)\right]\right. \\
& \left.-\sum_{j=1}^{n_{2}} \delta_{j-1}^{2} B_{t}\left(T_{j}^{2}\right) \mathbb{E}_{t}^{T_{j}^{2}}\left[L_{T_{j-1}^{2}}\left(T_{j-1}^{2}, T_{j}^{2}\right)+K\right]\right) .
\end{aligned}
$$


Thus,

$$
P_{t}^{b s w}=N\left(\sum_{i=1}^{n_{1}} B_{t}\left(T_{i}^{1}\right) F_{t}\left(T_{i-1}^{1}, T_{i}^{1}\right)-\sum_{j=1}^{n_{2}} B_{t}\left(T_{j}^{2}\right)\left(F_{t}\left(T_{j-1}^{2}, T_{j}^{2}\right)+\delta_{j-1}^{2} K\right)\right) .
$$

The value of the basis swap after the initiation, i.e. the value at time $t$, for $T_{0}^{1} \leq t<T_{n_{1}}^{1}$, is given by

$$
\begin{aligned}
P_{t}^{b s w}= & N\left(B_{t}\left(T_{i_{t}}^{1}\right) F_{T_{i_{t}-1}^{1}}\left(T_{i_{t}-1}^{1}, T_{i_{t}}^{1}\right)+\sum_{i=i_{t}+1}^{n_{1}} B_{t}\left(T_{i}^{1}\right) F_{t}\left(T_{i-1}^{1}, T_{i}^{1}\right)\right. \\
& \left.-B_{t}\left(T_{j_{t}}^{2}\right)\left(F_{T_{j_{t}-1}^{2}}\left(T_{j_{t}-1}^{2}, T_{j_{t}}^{2}\right)+\delta_{j_{t}-1}^{2} K\right)-\sum_{j=j_{t}+1}^{n_{2}} B_{t}\left(T_{j}^{2}\right)\left(F_{t}\left(T_{j-1}^{2}, T_{j}^{2}\right)+\delta_{j-1}^{2} K\right)\right)
\end{aligned}
$$

where $T_{i_{t}}^{1}$, respectively $T_{j_{t}}^{2}$, denotes the smallest $T_{i}^{1}$, respectively $T_{j}^{2}$, which is strictly greater than $t$.

The fair basis swap spread at time $t$, denoted by $K_{t}^{b s w}$, is the spread $K$ such that the value of the basis swap at time time is equal to zero, i.e. it is given by

$$
K_{t}^{b s w}=\frac{\sum_{i=1}^{n_{1}} B_{t}\left(T_{i}^{1}\right) F_{t}\left(T_{i-1}^{1}, T_{i}^{1}\right)-\sum_{j=1}^{n_{2}} B_{t}\left(T_{j}^{2}\right) F_{t}\left(T_{j-1}^{2}, T_{j}^{2}\right)}{\sum_{j=1}^{n_{2}} \delta_{j-1}^{2} B_{t}\left(T_{j}^{2}\right)} .
$$

\subsection{Caplets}

Let us now consider a caplet with strike $K$ and maturity $T$ on the spot Libor rate for the period $[T, T+\delta]$, settled in arrears at time $T+\delta$. Since

$$
L_{T}(T, T+\delta)=\mathbb{E}_{T}^{T+\delta}\left[L_{T}(T, T+\delta)\right]=\delta^{-1} F_{T}(T, T+\delta),
$$

the time- $t$ price of the caplet is given, for $t \leq T$, by

$$
\begin{aligned}
P_{t}^{c p l} & =\delta B_{t}(T+\delta) \mathbb{E}_{t}^{T+\delta}\left[\left(L_{T}(T, T+\delta)-K\right)^{+}\right] \\
& =B_{t}(T+\delta) \mathbb{E}_{t}^{T+\delta}\left[\left(F_{T}(T, T+\delta)-\delta K\right)^{+}\right] .
\end{aligned}
$$

In particular,

$$
P_{0}^{c p l}=B_{0}(T+\delta) \mathbb{E}^{T+\delta}\left[\left(e^{X}-\bar{K}\right)^{+}\right]
$$

where $\bar{K}:=\delta K$ and

$$
X:=\log F_{T}(T, T+\delta)=\log F_{0}(T, T+\delta)+\int_{0}^{T} \alpha(s, T, T+\delta) d s+\int_{0}^{T} \varsigma(s, T, T+\delta) d Y_{s}
$$

Denoting by $\psi\left(\right.$ resp. $\psi^{T+\delta}$ ) the cumulant generating function of the process $Y$ under the measure $\mathbb{P}$ (resp. $\mathbb{P}^{T+\delta}$ ), the moment generating function of the random variable $X$ under 
the measure $\mathbb{P}^{T+\delta}$ is given, for any $z \in \mathbb{R}$ such that the expectation below exists, by

$$
\begin{aligned}
M_{X}^{T+\delta}(z)=\mathbb{E}^{T+\delta}\left[e^{z X}\right] & \exp \left(z\left(\log F_{0}(T, T+\delta)+\int_{0}^{T} \alpha(s, T, T+\delta) d s\right)\right) \\
\quad & \times \exp \left(\int_{0}^{T} \psi_{s}^{T+\delta}(z \varsigma(s, T, T+\delta)) d s\right) \\
=\exp \left(z\left(\log F_{0}(T, T+\delta)+\int_{0}^{T} \alpha(s, T, T+\delta) d s\right)\right) & \\
& \times \exp \left(-\int_{0}^{T} \psi(-\Sigma(s, T+\delta)) d s\right) \\
& \times \exp \left(\int_{0}^{T} \psi(z \varsigma(s, T, T+\delta)-\Sigma(s, T+\delta)) d s\right),
\end{aligned}
$$

by Lemma 3.1 in Eberlein and Raible (1999) and (13). The payoff function of the caplet

$$
g(x)=\left(\mathrm{e}^{x}-\bar{K}\right)^{+},
$$

has the (generalized) Fourier transform

$$
\widehat{g}(z)=\int_{\mathbb{R}} e^{i z x} g(x) d x=\frac{\bar{K}^{1+i z}}{i z(1+i z)},
$$

for $z \in \mathbb{C}$ such that $\Im z>1$.

Proposition 3.2 Assume a positive constant $\widetilde{\mathcal{K}}<\frac{\mathcal{K}}{2}$ is such that $\Sigma(s, T) \leq \widetilde{\mathcal{K}}$ and $\varsigma(s, T, S) \leq$ $\widetilde{\mathcal{K}}$, componentwise and for all $0 \leq s \leq T \leq S \leq \bar{T}$. For any $R \in\left(1, \frac{\mathcal{K}-\widetilde{\mathcal{K}}}{\widetilde{\mathcal{K}}}\right)$,

$$
\begin{aligned}
P_{0}^{c p l} & =\frac{B_{0}(T+\delta)}{2 \pi} \int_{\mathbb{R}} \widehat{g}(i R-v) M_{X}^{T+\delta}(R+i v) d v \\
& =\frac{B_{0}(T+\delta)}{2 \pi} \int_{\mathbb{R}} \frac{\bar{K}^{1-\mathrm{i} v-R} M_{X}^{T+\delta}(R+\mathrm{i} v)}{(R+\mathrm{i} v)(R+\mathrm{i} v-1)} d v .
\end{aligned}
$$

Proof. The result follows by Theorem 2.2 in Eberlein, Glau, and Papapantoleon (2010). We only have to check that $M_{X}^{T+\delta}(R+\mathrm{i} v)$ is finite, which follows from $\mid R \varsigma^{i}(s, T, T+\delta)-$ $\Sigma^{i}(s, T+\delta) \mid<\mathcal{K}$, for $R \in\left(1, \frac{\mathcal{K}-\widetilde{\mathcal{K}}}{\widetilde{\mathcal{K}}}\right)$ and every $i=1, \ldots, n$.

\subsection{Swaptions}

In this section we consider a swaption, which is an option to enter an interest rate swap with swap rate $K$ and maturity $T_{n}$ at a pre-specified date $T=T_{0} \geq 0$. The underlying swap is defined in Sect. 3 and we consider the notional amount $N=1$. The swaption can be seen as a sequence of fixed payments $\delta_{j-1}\left(K_{T}^{s w}-K\right)^{+}, j=1, \ldots, n$, received at dates $T_{1}, \ldots, T_{n}$, where $K_{T}^{s w}$ denotes the swap rate of the underlying swap at time $T$. Thus, the value at time $t \leq T$ of the swaption is given by

$$
P_{t}^{s w n}=B_{t}(T) \sum_{j=1}^{n} \delta_{j-1} \mathbb{E}_{t}^{T}\left[B_{T}\left(T_{j}\right)\left(K_{T}^{s w}-K\right)^{+}\right]
$$


In particular,

$$
\begin{aligned}
P_{0}^{s w n} & =B_{0}(T) \mathbb{E}^{T}\left[\sum_{j=1}^{n} \delta_{j-1} B_{T}\left(T_{j}\right)\left(K_{T}^{s w}-K\right)^{+}\right] \\
& =B_{0}(T) \mathbb{E}^{T}\left[\left(\sum_{j=1}^{n} B_{T}\left(T_{j}\right) F_{T}\left(T_{j-1}, T_{j}\right)-\sum_{j=1}^{n} \delta_{j-1} B_{T}\left(T_{j}\right) K\right)^{+}\right]
\end{aligned}
$$

by (15). To proceed with the computation of the above expectation, we shall impose certain assumptions on the volatility structures $\Sigma(t, T)$ and $\varsigma(t, T, S)$. First, we assume that

$$
\frac{\partial}{\partial T} \Sigma(t, T)=\sigma^{1}(t) \sigma^{2}(T)
$$

where $\sigma^{1}:[0, \bar{T}] \rightarrow \mathbb{R}^{n}$ and $\sigma^{2}:[0, \bar{T}] \rightarrow \mathbb{R}$. This separable volatility assumption implies that

$$
\Sigma(t, T)-\Sigma(t, S)=\Sigma^{1}(t) \Sigma^{2}(T, S)
$$

where $\Sigma^{1}:[0, \bar{T}] \rightarrow \mathbb{R}^{n}$ and $\Sigma^{2}:[0, \bar{T}] \times[0, \bar{T}] \rightarrow \mathbb{R}$. For the volatilities $\varsigma(t, T, S)$, we assume similarly

$$
\varsigma(t, T, S)=\varsigma^{1}(t) \varsigma^{2}(T, S),
$$

where $\varsigma^{1}:[0, \bar{T}] \rightarrow \mathbb{R}^{n}$ and $\varsigma^{2}:[0, \bar{T}] \times[0, \bar{T}] \rightarrow \mathbb{R}$. Let

$$
Z=\left(Z^{1}, Z^{2}\right)=\left(\int_{0}^{T} \Sigma^{1}(t) d Y_{t}, \int_{0}^{T} \varsigma^{1}(t) d Y_{t}\right)
$$

Recalling (6), we obtain for each $j$

$$
\begin{aligned}
B_{T}\left(T_{j}\right) & =\frac{B_{0}\left(T_{j}\right)}{B_{0}(T)} \exp \left(\int_{0}^{T}\left(A(s, T)-A\left(s, T_{j}\right)\right) d s+\int_{0}^{T}\left(\Sigma(s, T)-\Sigma\left(s, T_{j}\right)\right) d Y_{s}\right) \\
& =c^{j, 0} e^{c^{j} Z^{1}} .
\end{aligned}
$$

Similarly, recalling (11), it follows for each $j$

$$
\begin{aligned}
F_{T}\left(T_{j-1}, T_{j}\right) & =F_{0}\left(T_{j-1}, T_{j}\right) \exp \left(\int_{0}^{T} \alpha\left(s, T_{j-1}, T_{j}\right) d s+\int_{0}^{T} \varsigma\left(s, T_{j-1}, T_{j}\right) d Y_{s}\right) \\
& =\bar{c}^{j, 0} e^{\bar{c}^{j} Z^{2}},
\end{aligned}
$$

where

$$
\begin{aligned}
c^{j, 0} & =\frac{B_{0}\left(T_{j}\right)}{B_{0}(T)} \exp \left(\int_{0}^{T}\left(A(s, T)-A\left(s, T_{j}\right)\right) d s\right), \\
c^{j} & =\Sigma^{2}\left(T, T_{j}\right), \\
\bar{c}^{j, 0} & =F_{0}\left(T_{j-1}, T_{j}\right) \exp \left(\int_{0}^{T} \alpha\left(s, T_{j-1}, T_{j}\right) d s\right), \\
\bar{c}^{j} & =\varsigma^{2}\left(T_{j-1}, T_{j}\right)
\end{aligned}
$$


are deterministic constants. Therefore, 25) reduces to

$$
P_{0}^{s w n}=B_{0}(T) \mathbb{E}^{T}\left[\left(\sum_{j=1}^{n} c^{j, 0} e^{c^{j} Z^{1}} \bar{c}^{j, 0} e^{\bar{c}^{j} Z^{2}}-\sum_{j=1}^{n} c^{j, 0} \delta_{j-1} K e^{c^{j} Z^{1}}\right)^{+}\right],
$$

which can be computed using the Fourier transform method (cf. Theorem 3.2 in Eberlein, Glau, and Papapantoleon (2010)). The value of the expectation depends only on the distribution of the random vector $Z$ under the measure $\mathbb{P}^{T}$, with moment generating function, for any $z \in \mathbb{R}^{2}$ such that the expectation below is finite, given by:

$$
\begin{aligned}
M_{Z}^{T}(z) & =\mathbb{E}^{T}\left[e^{z_{1} Z^{1}+z_{2} Z^{2}}\right] \\
& =\mathbb{E}^{T}\left[e^{\int_{0}^{T} z_{1} \Sigma^{1}(s) d Y_{s}+\int_{0}^{T} z_{2} \varsigma^{1}(s) d Y_{s}}\right] \\
& =\exp \left(\int_{0}^{T} \psi_{s}^{T}\left(z_{1} \Sigma^{1}(s)+z_{2} \varsigma^{1}(s)\right) d s\right) \\
& =\exp \left(-\int_{0}^{T} \psi(-\Sigma(s, T)) d s\right) \exp \left(\int_{0}^{T} \psi\left(\left(z_{1} \Sigma^{1}(s)+z_{2} \varsigma^{1}(s)\right)-\Sigma(s, T)\right) d s\right)
\end{aligned}
$$

by Lemma 3.1 in Eberlein and Raible (1999) and (13). Let

$$
f(x)=f\left(x_{1}, x_{2}\right):=\left(\sum_{j=1}^{n} c^{j, 0} \bar{c}^{j, 0} e^{c^{j} x_{1}+\bar{c}^{j} x_{2}}-\sum_{j=1}^{n} c^{j, 0} \delta_{j-1} K e^{c^{j} x_{1}}\right)^{+}
$$

with (generalized) Fourier transform

$$
\widehat{f}(z)=\int_{\mathbb{R}^{2}} e^{i z x} f(x) d x
$$

for any row-vector $z \in \mathbb{C}^{2}$ such that the above integral is finite (see Remark 3.4 and Hubalek and Kallsen (2005), Hurd and Zhou (2010) for more details on the computation of $f$ ). The following result follows directly from Eberlein, Glau, and Papapantoleon (2010, Theorem 3.2) applied to $(33)$.

Proposition 3.3 The time-0 price of the swaption is given by

$$
P_{0}^{s w n}=\frac{B_{0}(T)}{(2 \pi)^{2}} \int_{\mathbb{R}^{2}} M_{Z}^{T}(R+\mathrm{i} u) \widehat{f}(\mathrm{i} R-u) d u,
$$

for any row-vector $R \in \mathbb{R}^{2}$ such that $M_{Z}^{T}(R+\mathrm{i} u)$ exists and the function $g(x):=e^{-R x} f(x)$ satisfies the prerequisites of Theorem 3.2 in Eberlein, Glau, and Papapantoleon (2010).

Remark 3.4 As opposed to the caplet Fourier formula (24), which can be readily implemented using one-dimensional FFT, the corresponding swaption Fourier formula $(36)$ is not so practical. First, computing $\widehat{f}$ in $(35)$ involves integrating an oscillating function in dimension two. Since $\widehat{f}$ itself is also highly oscillatory we end up with a significant computational burden in calculating the two-dimensional integral in (36). However, swaptions can 
instead be efficiently valued numerically based on (33), rewritten in the notation introduced above as:

$$
\begin{aligned}
P_{0}^{s w n}=B_{0}(T)\left(\sum_{j=1}^{n} c^{j, 0} \bar{c}^{j, 0} \mathbb{E}^{T}\left[e^{c^{j} Z^{1}+\bar{c}^{j} Z^{2}} \mathbb{1}_{\{\widetilde{f}(Z) \geq 0\}}\right]\right. \\
\left.-\sum_{j=1}^{n} c^{j, 0} \delta_{j-1} K \mathbb{E}^{T}\left[e^{c^{j} Z^{1}} \mathbb{1}_{\{\widetilde{f}(Z) \geq 0\}}\right]\right) .
\end{aligned}
$$

Here the function $\tilde{f}$ is given by

$$
\widetilde{f}\left(x_{1}, x_{2}\right)=\sum_{j=1}^{n} c^{j, 0} \bar{c}^{j, 0} e^{c^{j} x_{1}+\bar{c}^{j} x_{2}}-\sum_{j=1}^{n} c^{j, 0} \delta_{j-1} K e^{c^{j} x_{1}} .
$$

Using (34) the expectations in (37) can be calculated using the standard methodology of Duffie, Pan, and Singleton (2000) after having replaced $\widetilde{f}$ with a linear approximation in the domain of integration $\{\tilde{f} \geq 0\}$. This method, known as the linear boundary approximation, is described in Singleton and Umantsev (2002).

\subsection{Lévy Hull-White Specification}

In the sequel we focus on the case where $Y=\left(Y^{1}, Y^{2}\right)$ is a two-dimensional Lévy process. We choose Vasicek volatility structures for $B_{t}(T)$ and $F_{t}(T, S)$, namely

$$
\begin{gathered}
\Sigma(s, T)=\left(\frac{\sigma}{a}\left(1-e^{-a(T-s)}\right), 0\right) \\
\varsigma(s, T, S)=\left(\frac{\sigma}{a} e^{a s}\left(e^{-a T}-e^{-a S}\right), \frac{\sigma^{*}(T, S)}{a^{*}} e^{a^{*} s}\left(e^{-a^{*} T}-e^{-a^{*} S}\right)\right),
\end{gathered}
$$

where $\sigma, \sigma^{*}(T, S)>0$ and $a, a^{*} \neq 0$ are real constants (see Remark 2.1). Note that the first component of the volatility $\varsigma(s, T, S)$ is exactly of the form $\Sigma^{1}(s, S)-\Sigma^{1}(s, T)$, thus offering an easy connection to the single-curve case discussed in Remark 2.1. Denote by $f_{t}(T)=-\partial_{T} \log \left(B_{t}(T)\right)$ the instantaneous continuously compounded forward rate, so that $r_{t}=f_{t}(t)$. The OIS bond price can be written in exponential-affine form as

$$
B_{t}(T)=\exp \left(m(t, T)+n(t, T) r_{t}\right)
$$

where

$$
\begin{aligned}
m(t, T)= & \log \left(\frac{B_{0}(T)}{B_{0}(t)}\right)-n(t, T)\left[f_{0}(t)+\psi^{1}\left(\frac{\sigma}{a}\left(e^{-a t}-1\right)\right)\right] \\
& -\int_{0}^{t}\left[\psi^{1}\left(\frac{\sigma}{a}\left(e^{-a(T-s)}-1\right)\right)-\psi^{1}\left(\frac{\sigma}{a}\left(e^{-a(t-s)}-1\right)\right)\right] d s
\end{aligned}
$$

and

$$
n(t, T)=-e^{a t} \int_{t}^{T} e^{-a u} d u=\frac{1}{a}\left(e^{-a(T-t)}-1\right) .
$$

The short rate $r$ is given by the following Lévy Hull-White extended Vasicek model (cf. Eberlein and Raible (1999), equation (4.11), and Example 3.5 of Crépey et al. (2012)):

$$
d r_{t}=a\left(\rho(t)-r_{t}\right) d t+\sigma d Y_{t}^{1}
$$


i.e.

$$
r_{t}=e^{-a t}\left(r_{0}+a \int_{0}^{t} e^{a s} \rho(s) d s+\sigma \int_{0}^{t} e^{a s} d Y_{s}^{1}\right)
$$

with

$$
\rho(t)=f_{0}(t)+\frac{1}{a} \frac{\partial}{\partial t} f_{0}(t)+\psi^{1}\left(\frac{\sigma}{a}\left(e^{-a t}-1\right)\right)-\left(\psi^{1}\right)^{\prime}\left(\frac{\sigma}{a}\left(e^{-a t}-1\right)\right) \frac{\sigma}{a} e^{-a t} .
$$

Similarly, the FRA rate given in (11) can be written as

$$
\begin{aligned}
F_{t}(T, S)= & F_{0}(T, S) \exp \left(\int_{0}^{t} \alpha(s, T, S) d s+\frac{\sigma}{a}\left(e^{-a T}-e^{-a S}\right) \int_{0}^{t} e^{a s} d Y_{s}^{1}\right. \\
& \left.+\frac{\sigma^{*}(T, S)}{a^{*}}\left(e^{-a^{*} T}-e^{-a^{*} S}\right) \int_{0}^{t} e^{a^{*} s} d Y_{s}^{2}\right) \\
= & \exp \left(m(t, T, S)+n(t, T, S) r_{t}+n^{*}(t, T, S) q_{t}\right),
\end{aligned}
$$

where

$$
\begin{aligned}
& m(t, T, S)=\log \left(F_{0}(T, S)\right)+\int_{0}^{t} \alpha(s, T, S) d s-n(t, T, S)\left(f_{0}(t)+\psi^{1}\left(\frac{\sigma}{a}\left(e^{-a t}-1\right)\right)\right) \\
& n(t, T, S)=n(t, T)-n(t, S) \\
& n^{*}(t, T, S)=\frac{\sigma^{*}(T, S)}{a^{*}}\left(e^{-a^{*}(T-t)}-e^{-a^{*}(S-t)}\right)
\end{aligned}
$$

and

$$
q_{t}=e^{-a^{*} t} \int_{0}^{t} e^{a^{*} s} d Y_{s}^{2}
$$

i.e.

$$
d q_{t}=-a^{*} q_{t} d t+d Y_{t}^{2}, \quad q_{0}=0 .
$$

Remark 3.5 The volatilities (38) and (39) satisfy separability conditions similar to (27) and (28), namely

$$
\begin{aligned}
\Sigma(t, T)-\Sigma(t, S) & =\frac{\sigma}{a}\left(e^{-a S}-e^{-a T}\right)\left(e^{a t}, 0\right) \\
\varsigma(t, T, S) & =\frac{\sigma}{a}\left(e^{-a T}-e^{-a S}\right)\left(e^{a t}, 0\right)+\frac{\sigma^{*}(T, S)}{a^{*}}\left(e^{-a^{*} T}-e^{-a^{*} S}\right)\left(0, e^{a^{*} t}\right) .
\end{aligned}
$$

The condition on $\Sigma(t, T)$ is exactly of the form (27) and the condition on $\varsigma(t, T, S)$ is slightly more general than (28), namely

$$
\varsigma(t, T, S)=\left(\varsigma^{1,1}(t) \varsigma^{2,1}(T, S), \varsigma^{1,2}(t) \varsigma^{2,2}(T, S)\right),
$$

where $\varsigma^{1,1}, \varsigma^{1,2}:[0, \bar{T}] \rightarrow \mathbb{R}$ and $\varsigma^{2,1}, \varsigma^{2,2}:[0, \bar{T}] \times[0, \bar{T}] \rightarrow \mathbb{R}$ are deterministic functions. Along the lines of the computations done in Sect. 3.3, the above assumptions enable us to express all quantities needed to price a swaption in terms of the random vector $\widetilde{Z}=$ $\left(\widetilde{Z}^{1}, \widetilde{Z}^{2}\right)=\left(\int_{0}^{T} e^{a s} d Y_{t}^{1}, \int_{0}^{T} e^{a^{*} s} d Y_{t}^{2}\right)$. We obtain the following counterpart of (33):

$$
P_{0}^{s w n}=B_{0}(T) \mathbb{E}^{T}\left[\left(\sum_{j=1}^{n} c^{j, 0} e^{\left(c^{j}+\bar{c}^{j, 1}\right) Z^{1}} \bar{c}^{j, 0} e^{\bar{c}^{j, 2} Z^{2}}-\sum_{j=1}^{n} c^{j, 0} \delta_{j-1} K e^{c^{j} Z^{1}}\right)^{+}\right],
$$


where

$$
\begin{aligned}
c^{j, 0} & =\frac{B_{0}\left(T_{j}\right)}{B_{0}(T)} \exp \left(\int_{0}^{T}\left(A(s, T)-A\left(s, T_{j}\right)\right) d s\right), \\
c^{j} & =\frac{\sigma}{a}\left(e^{-a T_{j}}-e^{-a T}\right), \\
\bar{c}^{j, 0} & =F_{0}\left(T_{j-1}, T_{j}\right) \exp \left(\int_{0}^{T} \alpha\left(s, T_{j-1}, T_{j}\right) d s\right), \\
\bar{c}^{j, 1} & =\frac{\sigma}{a}\left(e^{-a T_{j-1}}-e^{-a T_{j}}\right), \\
\bar{c}^{j, 2} & =\frac{\sigma^{*}\left(T_{j-1}, T_{j}\right)}{a^{*}}\left(e^{-a^{*} T_{j-1}}-e^{-a^{*} T_{j}}\right)
\end{aligned}
$$

are deterministic constants. The relevant function $f$ in an analog of Proposition 3.3 is given by

$$
f(x)=f\left(x_{1}, x_{2}\right):=\left(\sum_{j=1}^{n} c^{j, 0} e^{\left(c^{j}+\bar{c}^{j, 1}\right) x_{1}} \bar{c}^{j, 0} e^{\bar{c}^{j, 2} x_{2}}-\sum_{j=1}^{n} c^{j, 0} \delta_{j-1} K e^{c^{j} x_{1}}\right)^{+}
$$

and the moment generating function of $\widetilde{Z}$ under the measure $\mathbb{P}^{T}$, denoted by $M_{\widetilde{Z}}^{T}(z)$, is given by

$$
\begin{aligned}
M_{\widetilde{Z}}^{T}(z)= & \mathbb{E}^{T}\left[e^{z_{1} \widetilde{Z}^{1}+z_{2} \widetilde{Z}^{2}}\right] \\
=\exp & \left(-\int_{0}^{T} \psi\left(\frac{\sigma}{a}\left(e^{-a(T-s)}-1\right), 0\right) d s\right) \\
& \quad \times \exp \left(\int_{0}^{T} \psi\left(z_{1} e^{a s}-\frac{\sigma}{a}\left(1-e^{-a(T-s)}\right), z_{2} e^{a^{*} s}\right) d s\right),
\end{aligned}
$$

for any $z \in \mathbb{R}^{2}$ such that the expectation above is finite. For numerical purposes, the linear boundary approximation method is preferred for the same reason as explained in Remark 3.4 .

Remark 3.6 Under the present assumptions, we can reformulate the expression for the time- 0 value of the caplet in Proposition 3.2 in terms of the factor process $(r, q)$ as

$$
P_{0}^{c p l}=B_{0}(T+\delta) \mathbb{E}^{T+\delta}\left[\left(e^{m(T, T, T+\delta)+n(T, T, T+\delta) r_{T}+n^{*}(T, T, T+\delta) q_{T}}-\bar{K}\right)^{+}\right]
$$

where we have used 42 . Similarly, the time-0 value of the swaption can be expressed as

$$
\begin{aligned}
P_{0}^{s w n}= & B_{0}(T) \mathbb{E}^{T}\left[\left(\sum_{j=1}^{n} e^{m\left(T, T_{j}\right)+n\left(T, T_{j}\right) r_{T}} e^{m\left(T, T_{j-1}, T_{j}\right)+n\left(T, T_{j-1}, T_{j}\right) r_{T}+n^{*}\left(T, T_{j-1}, T_{j}\right) q_{T}}\right.\right. \\
& \left.\left.-\sum_{j=1}^{n} \delta_{j-1} K e^{m\left(T, T_{j}\right)+n\left(T, T_{j}\right) r_{T}}\right)^{+}\right] \\
= & B_{0}(T) \mathbb{E}^{T}\left[\left(\sum_{j=1}^{n} \alpha^{j, 0} e^{\alpha^{j, 1} r_{T}+\alpha^{j, 2} q_{T}}-\sum_{j=1}^{n} \beta^{j, 0} e^{\beta^{j, 1} r_{T}}\right)^{+}\right]
\end{aligned}
$$


where we have used (40) and 42). Here $\alpha^{j, 0}=e^{m\left(T, T_{j}\right)+m\left(T, T_{j-1}, T_{j}\right)}, \alpha^{j, 1}=n\left(T, T_{j}\right)+$ $n\left(T, T_{j-1}, T_{j}\right), \alpha^{j, 2}=n^{*}\left(T, T_{j-1}, T_{j}\right), \beta^{j, 0}=e^{m\left(T, T_{j}\right)} \delta_{j-1} K$ and $\beta^{j, 1}=n\left(T, T_{j}\right)$. One can now proceed as in Proposition 3.3 and obtain a Fourier pricing formula alternative to (36).

\subsection{Markovian Perspective}

The above Lévy Hull-White model produces the following two-dimensional factor process $\left(r_{t}, q_{t}\right)$ driven by two $(\mathbb{F}, \mathbb{P})$ Lévy processes $Y^{1}$ and $Y^{2}$ :

$$
\begin{aligned}
& d r_{t}=a\left(\rho(t)-r_{t}\right) d t+\sigma_{1} d Y_{t}^{1}, \quad r_{0}=\text { const. } \\
& d q_{t}=-a^{*} q_{t} d t+d Y_{t}^{2}, \quad q_{0}=0 .
\end{aligned}
$$

In view of 40 and $(42)$, the price $B_{t}(T)$ of the OIS bond (resp. the FRA rate $F_{t}(T, S)$ ) can be written as an exponential-affine function of $r_{t}$ (resp. of $r_{t}, q_{t}$ ). To price linear payoffs, one simply has to insert the above representations for $B_{t}(T)$ and $F_{t}(T, S)$ into corresponding equations for each product. Consequently, all these prices can be represented by explicit formulas of the form

$$
P_{t}=P\left(t, X_{t}\right), \quad t \in[0, \bar{T}],
$$

where $P$ is a deterministic function and $X_{t}$ is a relevant Markovian factor process. For instance, in the case of the basis swap on which TVA computations will be performed in Sect. 5.2 equation 20 yields $X_{t}=\left(r_{t}, q_{t}, r_{t}^{1}, q_{t}^{1}, r_{t}^{2}, q_{t}^{2}\right)$, where

$$
r_{t}^{1}=r_{T_{i_{t}-1}}, \quad q_{t}^{1}=q_{T_{i_{t}-1}}, \quad r_{t}^{2}=r_{T_{j_{t}-1}}, q_{t}^{2}=q_{T_{j_{t}-1}},
$$

which is a six-dimensional Markovian factor process. The path-dependence which is reflected by the last four factors in $X_{t}$ is due to the fact that both legs of the basis swap deliver payments in arrears.

Remark 3.7 Going back to the general setup of Sect.2.2 and under the separable volatility assumptions (26) and (28) from Sect. 3.3, similar two-factor Markovian representations of $P_{t}$ can be obtained as well, in terms of the Markovian short rate process $r_{t}$ and of a second Markovian factor given as $Z_{t}^{2}:=\int_{0}^{t} \varsigma^{1}(s) d Y_{s}$.

Remark 3.8 Deterministic OIS rates could also be considered without major inconsistency in the model since, for lack of liquid OIS option data, the OIS volatility parameters cannot be identified from the market anyway (see Sect. 4.3 regarding the way we fix them in the numerical implementation). Deterministic OIS rates are obtained by letting $\sigma$ be zero in (38)-(39), which results in a one-factor Markov multiple-curve model $q_{t}$ driven by $Y^{2}$ (note that $Y^{1}$ plays no role when $\sigma$ is zero). The computations of the next sections were done also for this one-factor specification and we found very little difference in the results, except of course for the short rate process in the upper graph of Figure 5 , which collapses to the process mean function (black curve in this graph).

\section{Model Calibration}

Recalling that a TVA can be viewed as a long-term option on the underlying contracts, both smile and term structure volatility effects matter for TVA computations. Furthermore, in 
view of the targeted application to TVA computations on multiple-curve products in Sect. 5 , it is important to achieve calibration to instruments of both $3 \mathrm{~m}$ and $6 \mathrm{~m}$ underlying tenors (by far the most liquid tenors). Calibration to both tenors is limited by the instruments traded in the market, but Bloomberg provides indicative or extrapolated quotes for the full swaption cube for both $3 \mathrm{~m}$ and $6 \mathrm{~m}$ tenors constructed in an arbitrage-free manner described in Levin and Zhang (2014) (see also Kienitz (2013)). We therefore fit the model to actual and indicative quotes. Specifically, we calibrate the two-dimensional Lévy model from Sect. 3.4 to EUR market Bloomberg data of January 4, 2011: Eonia, 3m Euribor and $6 \mathrm{~m}$ Euribor initial term structures on the one hand, $3 \mathrm{~m}$ and $6 \mathrm{~m}$ tenor swaptions on the other hand. In the first step, we calibrate the non-maturity/tenor dependent parameters to the swaption smile for the $9 y \times 1 y$ swaption with a $3 \mathrm{~m}$ tenor underlying. This also gives us the values of $\sigma^{*}(9,9.25), \sigma^{*}(9.25,9.5), \sigma^{*}(9.5,9.75)$ and $\sigma^{*}(9.75,10)$. In the second step, we use at-the-money swaptions on $3 \mathrm{~m}$ and $6 \mathrm{~m}$ tenor swaps all terminating at exactly 10 years, but with maturities from 1 to 9 years. This co-terminal procedure is chosen with a view towards the TVA application in Sect. 5, where a basis swap with a 10 year terminal date, and then a swaption on it, are considered.

Note that market quotes typically reflect prices of fully collateralized transactions, which can be considered as clean prices (see Sect. 3.3 in Crépey et al. (2013)). The clean price of the previous section is thus the relevant notion of valuation at the stage of model calibration.

\subsection{Choice of the Driving Process}

We consider a two-dimensional Lévy process $Y=\left(Y^{1}, Y^{2}\right)$ with independent components

$$
Y_{t}^{i}=L_{t}^{i}+\sum_{j=1}^{N_{t}^{i}} J_{j}^{i}, \quad i=1,2,
$$

where:

- $L^{i}$ is a finite moment $\log$ stable process (FMLS), i.e. a Lévy $\alpha_{i}$-stable process with tail index $\alpha_{i} \in(0,2]$, location parameter zero and the maximum negative skewness parameter $\beta_{i}=-1$,

- the sum is a Merton-like jump component, i.e. $N^{i}$ is a Poisson process with intensity $\lambda_{i}$ and the $J_{j}^{i}, j \in \mathbb{N}$, are independent copies of the random variable $J^{i} \sim N\left(\bar{\mu}_{i}, \bar{\sigma}_{i}^{2}\right)$.

FMLS processes have been introduced for option pricing in Carr and Wu (2003). Briefly, they are a limiting case in the class of $\alpha$-stable Lévy processes with the maximum negative skewness, which ensures finite positive exponential moments of all orders, i.e.

$$
\mathbb{E}\left[\exp \left(u L_{t}^{i}\right)\right]<\infty, \quad \text { for all } u>0,
$$

a property that more commonly used Lévy drivers such as CGMY, NIG or VG do not possess. The process $Y^{i}$ satisfies condition (2) with $\mathcal{K}_{1}=\mathcal{K}_{2}=\infty$ if $\alpha_{i}=2$ and $\mathcal{K}_{1}=0$ and $\mathcal{K}_{2}=\infty$ if $\alpha_{i}<2$. The cumulant generating function of $Y^{i}$ is

$$
\psi^{i}\left(z_{i}\right)=-z_{i}^{\alpha_{i}} \sec \left(\pi \alpha_{i} / 2\right)+\lambda_{i}\left(\exp \left(\bar{\mu}_{i} z_{i}+\frac{1}{2} \bar{\sigma}_{i}^{2} z_{i}^{2}\right)-1\right),
$$


for $z_{i} \in \mathbb{R}$ such that $\mathcal{K}_{1} \leq z_{i} \leq \mathcal{K}_{2}$ (regarding the cumulant generating function of the FMLS component, see Carr and Wu (2003, equation (7))). In the sequel we use $\alpha_{1}=2$ and $\alpha_{2} \in(0,2)$ combined with the Vasicek volatility structures of Sect. 3.4. In other words, we set $L^{1} \sim \sqrt{2} W$, where $W$ is a standard Bownian motion, and $L^{2}$ an FMLS process with index $\alpha_{2}<2$. Thanks to (48), we do not have to set a priori any upper limit on the value of the volatility $\varsigma(s, T, S)$, which we have found to be an advantage in the calibration procedure when moving across the term structure, i.e. across different values of $T, S$. More precisely, the cumulant generating function $\psi^{1}\left(z_{1}\right)$ of $Y^{1}$ exists for any $z_{1} \in \mathbb{R}$ and the cumulant generating function $\psi^{2}\left(z_{2}\right)$ of $Y^{2}$ exists for any $z_{2} \geq 0$. Thus, inspection of formula (13) reveals that, due to the independence of $Y^{1}$ and $Y^{2}$, the cumulant generating function $\psi_{s}^{T}(z)$ of the process $Y$ under the forward measure $\mathbb{P}^{T}$ exists and is given by

$$
\psi_{s}^{T}\left(z_{1}, z_{2}\right)=\psi^{1}\left(z_{1}-\Sigma^{1}(s, T)\right)+\psi^{2}\left(z_{2}\right)-\psi^{1}\left(-\Sigma^{1}(s, T)\right),
$$

for any $z=\left(z_{1}, z_{2}\right) \in \mathbb{R}_{+}^{2}$. Hence, no upper limits are needed neither for the values of $\varsigma(s, T, S)$, nor of $\Sigma(s, T)$. The Brownian choice for the OIS-driving component above is made for pragmatic reasons, only in order to simplify and speed up the numerical procedures used in the calibration, and at no cost given the lack of liquid market data for nonlinear derivatives written on the OIS rate.

\subsection{Initial Term Structures}

The initial term structures of the Eonia, 3m Euribor and $6 \mathrm{~m}$ Euribor markets (and therefore also the initial values of $3 \mathrm{~m}-6 \mathrm{~m}$ basis swaps in view of $(19 p)$ are fitted automatically in the HJM setup. More precisely, Bloomberg provides zero coupon discount bond prices $B_{0}^{i}(T)$ and yields $R_{0}^{i}(T)=-\ln \left(B_{0}^{i}(T)\right) / T$ for any tenor $i=\mathrm{d}$, 3m and $6 \mathrm{~m}$ (where "d" stands for "one day" in reference to the OIS market) and for any maturity $T$, constructed in a manner described in Akkara (2012). For $i=3 \mathrm{~m}$, resp. 6m, in principle we only need a term structure of the $F_{0}\left(T, T+\delta_{i}\right)$, where $\delta_{i}=\frac{1}{4}$, resp. $\frac{1}{2}$, which can be extracted from the following formula based on Bloomberg synthetic $3 \mathrm{~m}$ and $6 \mathrm{~m}$ discount bond prices $B_{0}^{i}\left(T_{k}^{i}\right)$ :

$$
F_{0}\left(T_{k-1}^{i}, T_{k}^{i}\right)=\frac{B_{0}^{i}\left(T_{k-1}^{i}\right)}{B_{0}^{i}\left(T_{k}^{i}\right)}-1 .
$$

A common problem with a direct computation based on (50) is that a smooth $R^{i}(T)$ curve does not necessarily result in a smooth FRA curve (see for example Hagan and West (2006)). A procedure yielding regular FRA curves is preferred since it typically gives rise to a more stable calibration. To bypass this problem, before applying (50), we fit the data using the least squares method to the following Nelson-Siegel-Svensson parametrization, for $i=\mathrm{d}$, $3 \mathrm{~m}$ and $6 \mathrm{~m}$ :

$$
R_{0}^{i}(T)=\beta_{0}^{i}+\beta_{1}^{i}\left(\frac{1-e^{-T \lambda_{1}^{i}}}{T \lambda_{1}^{i}}\right)+\beta_{2}^{i}\left(\frac{1-e^{-T \lambda_{1}^{i}}}{T \lambda_{1}^{i}}-e^{-T \lambda_{1}^{i}}\right)+\beta_{3}^{i}\left(\frac{1-e^{-T \lambda_{2}^{i}}}{T \lambda_{2}^{i}}-e^{-T \lambda_{2}^{i}}\right) .
$$

The results are plotted in Figure 1, which corresponds to the following parameters:

\begin{tabular}{c|cccccc} 
& $\beta_{0}^{i}$ & $\beta_{1}^{i}$ & $\beta_{2}^{i}$ & $\beta_{3}^{i}$ & $\lambda_{1}^{i}$ & $\lambda_{2}^{i}$ \\
\hline Eonia & $1.042 \times 10^{-5}$ & 0.00319198 & 0.095675 & 0.02100 & 0.07264 & 0.08429 \\
3m Euribor & $7.8542 \times 10^{-} 4$ & 0.004575 & 0.2384 & 0.096819 & 0.0012073 & 0.10283 \\
6m Euribor & 0.007656 & $6.4046 \times 10^{-5}$ & 0.04434 & 0.092094 & 0.006675 & 0.10531
\end{tabular}



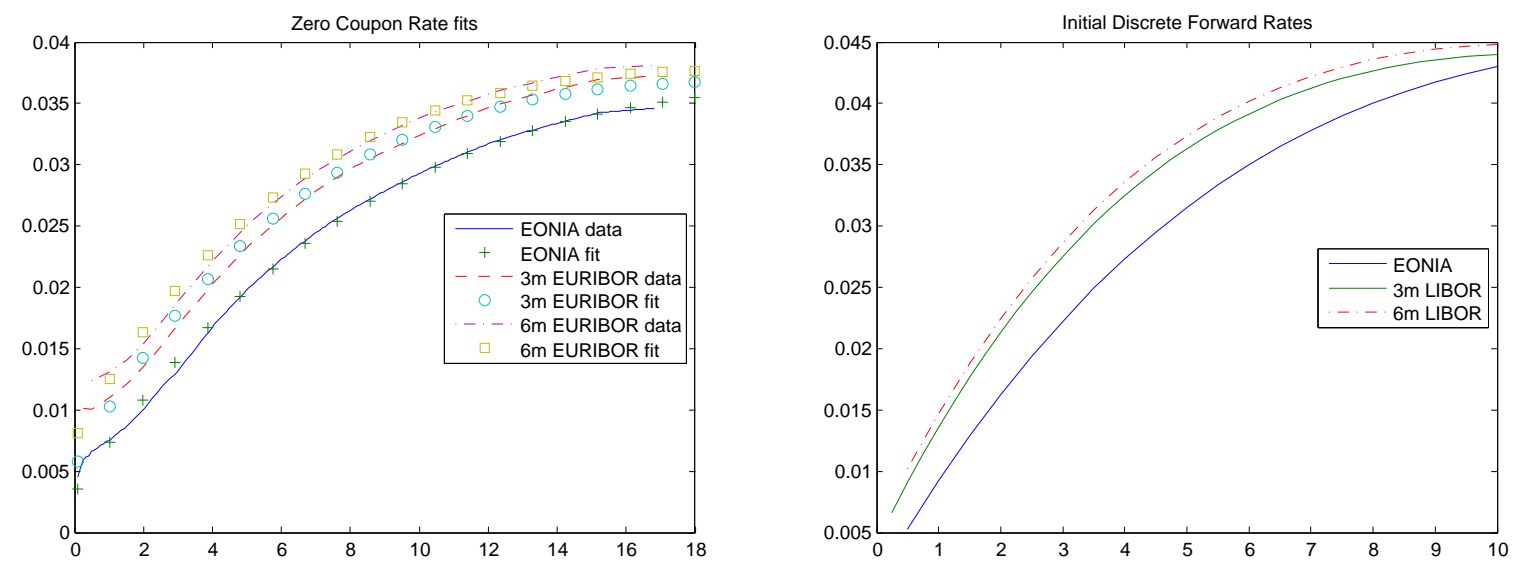

Figure 1: Initial term structures. Left: Zero coupon rates. Right: Discrete forward rates.

\subsection{Calibration to OTM Swaptions}

Next, we fix the following parameters in (38), (39) and (49):

$$
\sigma=0.008 / \sqrt{2}, \quad a=0.05, \quad a^{*}=0, \quad \alpha_{1}=2, \quad \lambda_{1}=0, \quad \bar{\mu}_{1}=0, \quad \bar{\sigma}_{1}=0 .
$$

As discussed in Sect. 4.1, the OIS curve is driven by a Brownian motion $\left(\alpha_{1}=2\right)$. The diffusion coefficient of this process is fixed at $\sigma \sqrt{2}=0.008$. Preliminary experiments revealed that $a^{*}$ does not have such effect in the end, apart from some (moderate) impact on the term structure of the swaption skew. Since we only calibrate the swaption skew at a single maturity, we set $a^{*}$ equal to zero for simplicity. Indeed, this assumption along with a Brownian driven OIS curve imply the following formula for the moment generating function in (44):

$$
\begin{aligned}
M_{\widetilde{Z}}^{T}(z)= & \exp \left(-\frac{\sigma^{2}\left(a T-\frac{3}{2}\right)}{a^{3}}-\frac{\sigma^{2} \mathrm{e}^{-2 a T}\left(4 \mathrm{e}^{a T}-1\right)}{2 a^{3}}\right) \\
& \times \exp \left(\frac{a\left(\sigma^{2} T-\sigma z_{1} \mathrm{e}^{a T}\right)-\frac{3 \sigma^{2}}{2}+\frac{a^{2} z_{1}{ }^{2} \mathrm{e}^{2 a T}}{2}}{a^{3}}+\frac{2 \sigma z_{1}+\frac{2 \sigma^{2} \mathrm{e}^{-a T}}{a}-\frac{\mathrm{e}^{-2 a T}\left(\sigma+a z_{1} \mathrm{e}^{a T}\right)^{2}}{2 a}}{a^{2}}\right) \\
& \times \exp \left(T \psi^{2}\left(z_{2}\right)\right)
\end{aligned}
$$

with $\psi^{2}$ as in $(49)$. Thanks to this formula, a swaption smile with 9 strikes can be calculated in a small fraction of a second and the full calibration procedure can be performed in a few minutes in Matlab. Note that the parameters in 52 were chosen to yield an implied volatility of $12 \%$ for the at-the-money-caplet written on the OIS discrete forward rate $\left(\frac{1}{B_{2.5}(3)}-1\right) / 0.5$. This implied volatility corresponds roughly to the implied volatility of the 3-year Euribor caplet, pre-crisis, when the OIS discrete forward rate levels were within a few basis points of the Euribor rate.

Regarding the remaining parameters, in the EUR swaption markets actual quotes are available for swap lengths of 2 years or more with $6 \mathrm{~m}$ tenor underlyings, and 1 year or less with $3 \mathrm{~m}$ tenor underlyings. We therefore calibrate the model to the $9 \mathrm{y} \times 1 \mathrm{y}$ swaption 
Figure 2: Market versus calibrated swaption implied volatilities.
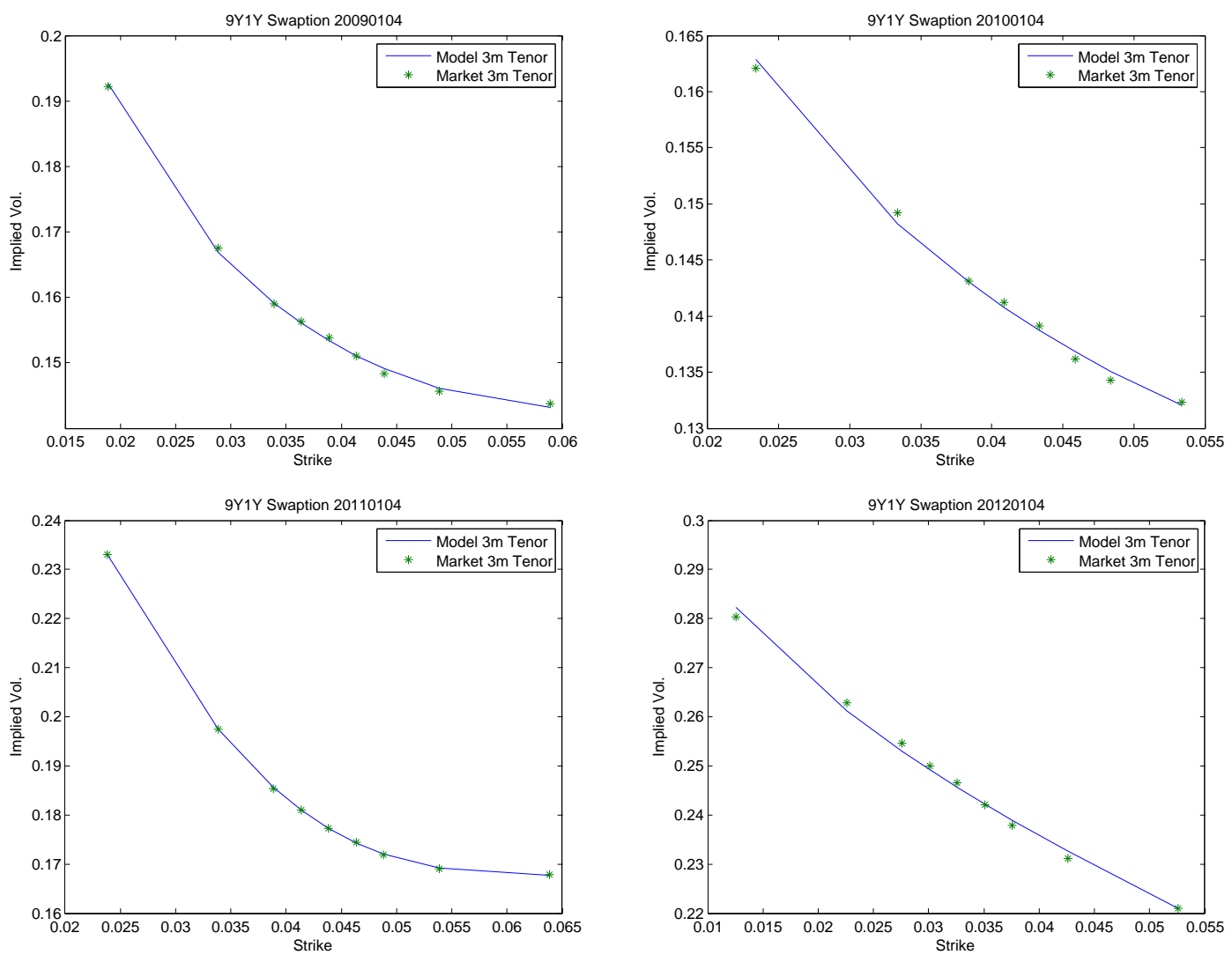

smile with a $3 \mathrm{~m}$ tenor underlying, using the linear boundary approximation method described in Remark 3.4 for pricing the swaption. This swaption has 4 payments and we simplify our calibration by setting the four parameters relating to those payments equal, i.e. $\sigma^{*}(9,9.25)=\cdots=\sigma^{*}(9.75,10)$. The following parameters are then obtained by least squares minimization to swaption market implied volatilities from January 4th 2011:

$$
\alpha_{2}=1.1117, \quad \lambda_{2}=0.0544, \quad \bar{\mu}_{2}=12.3518, \quad \bar{\sigma}_{2}=1.1078 \quad \sigma^{*}(9,9.25)=0.1259 .
$$

A value of $\alpha_{2}$ slightly above 1 corresponds to an infinite activity and variation, real valued process $L^{2}$. In order to illustrate the model capabilities we also calibrate to 3 other dates. As can be seen in Figure 2, the model fits very well to the observed market quotes throughout the entire crisis period.

\subsection{Calibration to Coterminal ATM Swaptions}

We proceed using the parameters found in the previous subsection and calibrate the remaining volatility parameters $\sigma^{*}(T, S)$ from a subset of $3 \mathrm{~m}$ and $6 \mathrm{~m}$ tenor at-the-money swaptions, priced by the linear boundary approximation method of Remark 3.4 .

We first consider $\Delta \times(10-\Delta)$ at-the-money swaptions with $\Delta=1 \mathrm{y}, 2 \mathrm{y}, \ldots, 9 \mathrm{y}$. These are available written on both $3 \mathrm{~m}$ and $6 \mathrm{~m}$ Euribor, but as stated in the previous section not all the quotes can be considered actual market quotes? 1 . We find the corresponding

\footnotetext{
${ }^{1}$ In this case only $9 \mathrm{y} \times 1 \mathrm{y}$ is quoted for a $3 \mathrm{~m}$ tenor, while the remaining maturities and swap lengths are
} 
$\sigma^{*}(T, S)$ parameters sequentially, starting with the $9 \mathrm{y} \times 1 \mathrm{y}$ swaption written on $6 \mathrm{~m}$ rates which contains two floating payments $L_{9}(9,9.5)$ and $L_{9.5}(9.5,10)$. We assume $\sigma^{*}(9,9.5)=$ $\sigma^{*}(9.5,10)$ and this common value is then found by calibrating to the at-the-money volatility of the $9 \mathrm{y} \times 1 \mathrm{y}$ swaption. Next, we move backward and calibrate to the $8 \mathrm{y} \times 2 \mathrm{y}$ swaption. We use the value of $\sigma^{*}(9,9.5)$ already determined and calibrate $\sigma^{*}(8,8.5)$ and $\sigma^{*}(8.5,9)$ by again assuming them equal. We continue in this manner down to the $1 \mathrm{y} \times 9 \mathrm{y}$ swaption, obtaining the values of $\sigma^{*}(T, T+0.5)=\sigma^{*}(T+0.5, T+1)$ for $T=1 \mathrm{y}, 2 \mathrm{y}, \ldots, 9 \mathrm{y}$. The short end volatility is extrapolated as $\sigma^{*}(0.5,1)=\sigma^{*}(1,1.5)$. For swaptions written on $3 \mathrm{~m}$ rates, we have four payments per year, hence four parameters to determine for each swaption. We reduce this to one parameter by assuming $\sigma^{*}(T, T+0.25)=\sigma^{*}(T+0.25, T+0.5)=\sigma^{*}(T+0.5, T+0.75)=$ $\sigma^{*}(T+0.75, T+1)$ for each $T=1 \mathrm{y}, 2 \mathrm{y}, \ldots, 8 \mathrm{y}$. The parameters are then determined sequentially, analogously to the $6 \mathrm{~m}$ case. Note that the procedure is started at the $8 \mathrm{y} \times 2 \mathrm{y}$ swaption since we have already determined the parameters related to the $9 \mathrm{y} \times 1 \mathrm{y}$ swaption smile in Sect. 4.3. The short end volatilities are again extrapolated as $\sigma^{*}(0.25,0.5)=$ $\sigma^{*}(0.5,0.75)=\sigma^{*}(0.75,1)=\sigma^{*}(1,1.25)$. The calibrated values of $\sigma^{*}(T, S)$ are plotted in the left panel of Figure 3 and the resulting market and model implied volatilities of the $\Delta \times$ $(10-\Delta)$ swaptions can be seen in the right panel. Note here that Bloomberg constructs the indicative market quotes of $3 \mathrm{~m}$ tenor volatility to be slightly higher than the corresponding $6 \mathrm{~m}$ tenor volatility. Figure 4 further shows that the linear boundary approximation method we use for swaption valuation is sufficiently accurate, comparing with implied volatilities obtained by an extensive Monte Carlo simulation using $m=10^{8}$ realizations. The level of the errors, although slightly larger for longer swap lengths (consistent with findings in Schrager and Pelsser (2006)), is economically insignificant. Other numerical experiments (not shown) verify that the approximation works equally well for other model parameters and swaption strikes, maturities and swap lengths. The computation time for estimating

Figure 3: Left: Calibrated values of $\sigma^{*}(T, S)$. Right: Implied volatility of the $\Delta \times(10-\Delta)$ co-terminal at-the-money swaptions
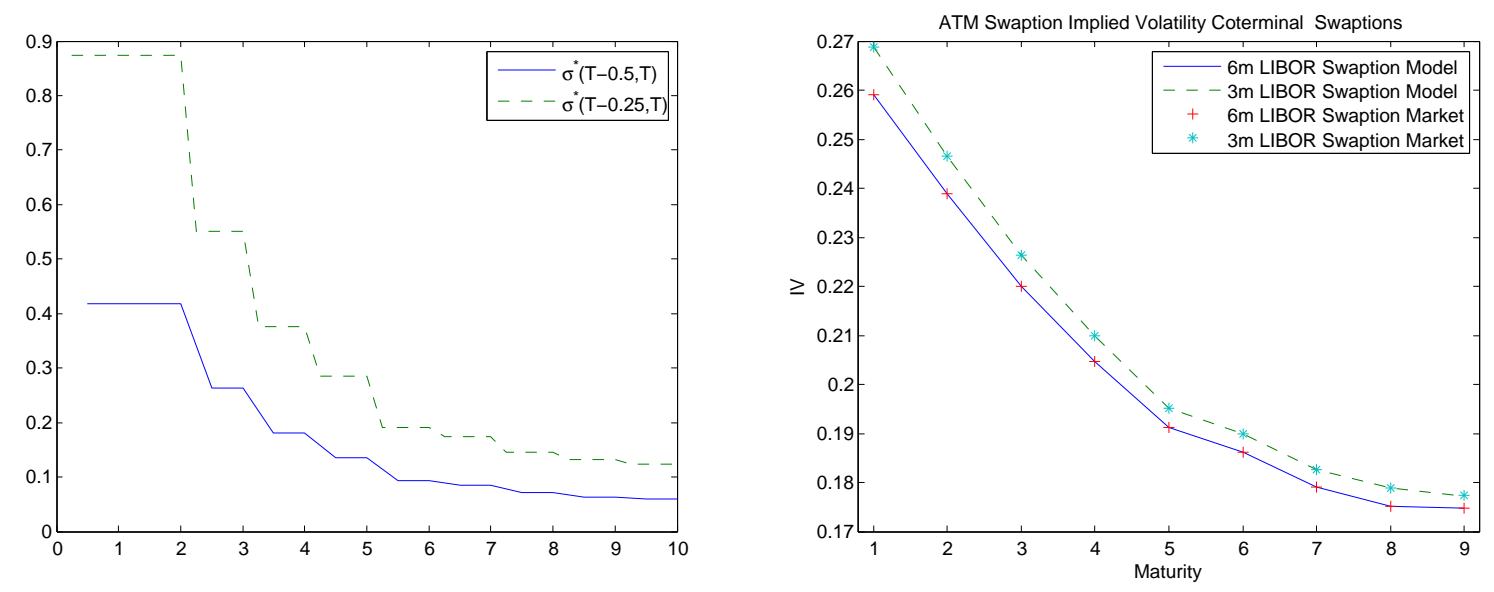

each expectation in formula (37) after applying the linear boundary approximation (see Remark 3.4 is roughly the one needed for valuing a caplet, which could be time-consuming.

quoted for $6 \mathrm{~m}$ tenor only. 
Figure 4: Errors due to the approximation in (37) calculated with calibrated parameters. The error is calculated as $10^{4} \times(\mathrm{MC}$ impld vol - linear boundary approx impld vol). Left: Implied volatility error in basis points of the $9 \mathrm{y} \times 1 \mathrm{y}$ swaption. Right: Implied volatility error in basis points of the $1 \mathrm{y} \times 9 \mathrm{y}$ swaption.
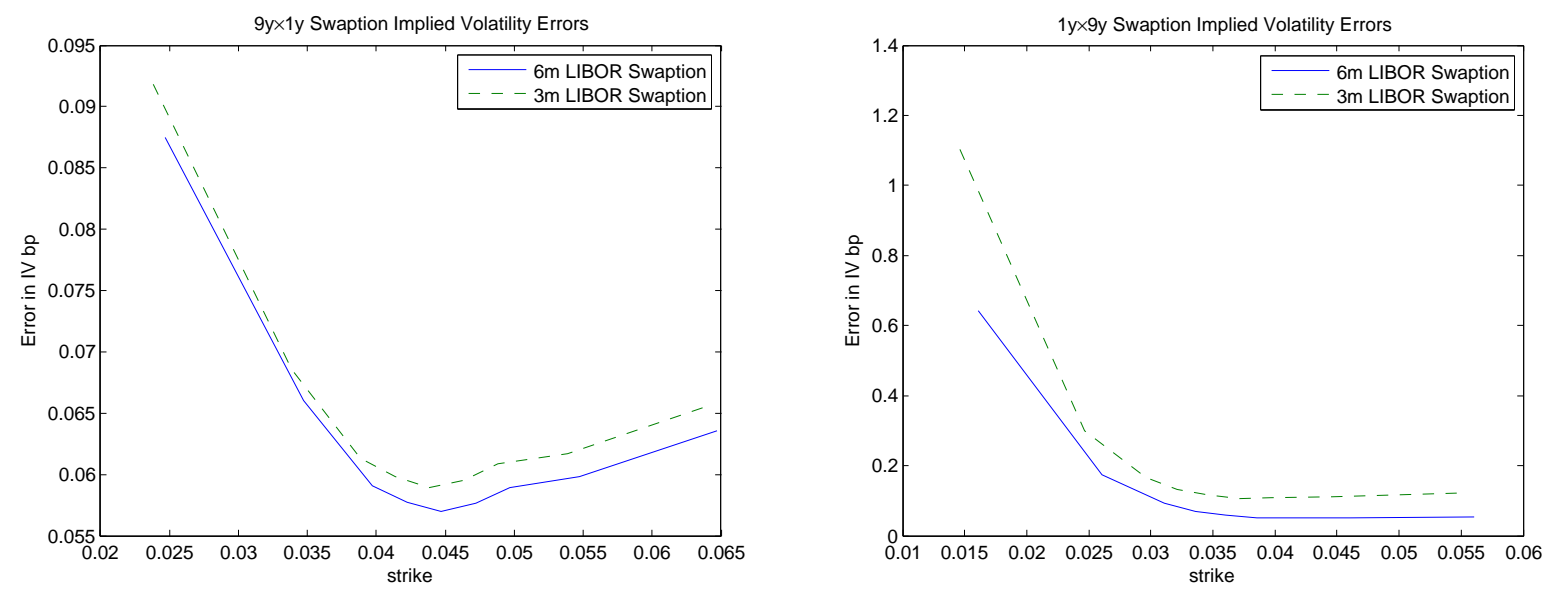

However, since we basically calibrate one $\sigma^{*}(T, S)$ parameter per swaption, our swaption calibration is quite fast.

\section{Counterparty Risk and Funding Adjustments}

In this section we follow the reduced-form methodology of Crépey (2012) (see also Pallavicini et al. (2012)), already used for the case of a single-curve interest rate model in Crépey et al. (2013), through which counterparty risk and funding adjustments are obtained as the solution to a related backward stochastic differential equation (BSDE). As illustration we study the case of a basis swap and a related swaption.

\subsection{TVA Equations}

Different interdependent valuation adjustments can be computed on top of the clean price $P$ in order to account for counterparty risk and funding constraints: credit/debt valuation adjustment CVA/DVA and liquidity funding valuation adjustment LVA, as well as replacement cost RC, with TVA for total valuation adjustment in aggregate, i.e.

$$
\mathrm{TVA}=\mathrm{CVA}+\mathrm{DVA}+\mathrm{LVA}+\mathrm{RC} .
$$

Each of these dependent terms has received a lot of attention in the recent literature. We refer to Sect. 1-3 of Crépey et al. (2013) and the references therein for details. Here we only recall that the TVA can be viewed as the price of an option on the "clean" value of the contract $P$ at the first-to-default time $\tau$ of a party. This option also pays dividends, which correspond to the funding benefit (in excess over the OIS rate $r_{t}$ ). Specifically, the TVA equation reads as the following backward stochastic differential equation (BSDE):

$$
\Theta_{t}=\mathbb{E}_{t}\left(\int_{t}^{\bar{T}} g_{s}\left(\Theta_{s}\right) d s\right), t \in[0, \bar{T}],
$$


where $\Theta_{t}, P_{t}$ and $g_{t}$ represent the TVA process, the clean price process and the counterparty risk and funding coefficient, respectively. The overall (selling) price of the contract for the bank (cost of the corresponding hedge, including the counterparty risk and funding features) is

$$
\Pi=P-\Theta .
$$

The coefficient $g_{t}$ in (55) is given, for every real $\vartheta$ (representing the TVA $\Theta_{t}$ that one is looking for in the probabilistic interpretation), by:

$$
\begin{aligned}
g_{t}(\vartheta)+r_{t} \vartheta= & \underbrace{-\gamma_{t}^{c}\left(1-\rho_{t}^{c}\right)\left(Q_{t}-\Gamma_{t}\right)^{-}}_{C V A \text { coeff. }}+\underbrace{\gamma_{t}^{b}\left(1-\rho_{t}^{b}\right)\left(Q_{t}-\Gamma_{t}\right)^{+}}_{D V A \text { coeff. }} \\
& +\underbrace{b_{t} \Gamma_{t}^{+}-\bar{b}_{t} \Gamma_{t}^{-}+\lambda_{t}\left(P_{t}-\vartheta-\Gamma_{t}\right)^{+}-\widetilde{\lambda}_{t}\left(P_{t}-\vartheta-\Gamma_{t}\right)^{-}}_{\text {LVA coeff. }} \\
& +\underbrace{\gamma_{t}\left(P_{t}-\vartheta-Q_{t}\right)}_{R C \text { coeff. }},
\end{aligned}
$$

where:

- $\gamma_{t}^{b}, \gamma_{t}^{c}$ and $\gamma_{t}$ are the default intensities of the bank, of its counterparty and their first-to-default intensity (in models where the bank and the counterparty can default together, $\gamma_{t}$ can be less than $\left.\gamma_{t}^{b}+\gamma_{t}^{c}\right)$,

- $\rho_{t}^{b}$ and $\rho_{t}^{c}$ are the recovery rates of the bank and the counterparty to each other,

- $Q_{t}$ is the value of the contract according to the scheme used by the liquidator in case $t=\tau<\bar{T}$, e.g. $Q_{t}=P_{t}$ (used henceforth unless otherwise stated) or $Q_{t}=P_{t}-\Theta_{t}$,

- $\Gamma_{t}=\Gamma_{t}^{+}-\Gamma_{t}^{-}$, where $\Gamma_{t}^{+}$(resp. $\Gamma_{t}^{-}$) is the value of the collateral posted by the bank to the counterparty (resp. by the counterparty to the bank), e.g. $\Gamma_{t}=0$ (used henceforth unless otherwise stated) or $\Gamma_{t}=Q_{t}$,

- $b_{t}$ and $\bar{b}_{t}$ are the spreads over the short rate $r_{t}$ for the remuneration of the collateral $\Gamma_{t}^{+}$and $\Gamma_{t}^{-}$posted by the bank and the counterparty to each other,

- $\lambda_{t}$ (resp. $\widetilde{\lambda}_{t}$ ) is the liquidity funding spread over the short rate $r_{t}$ corresponding to the remuneration of the external funding loan (resp. debt) of the bank. By liquidity funding spreads we mean that these are free from credit risk, i.e.

$$
\widetilde{\lambda}_{t}=\bar{\lambda}_{t}-\gamma_{t}^{b}\left(1-\mathfrak{r}^{b}\right)
$$

where $\bar{\lambda}_{t}$ is the funding borrowing spread (all inclusive) of the bank and $\mathfrak{r}^{b}$ stands for a recovery rate of the bank to its unsecured funder. In the case of $\lambda_{t}$ there is no credit risk involved anyway since the funder of the bank is assumed risk-free.

The data $Q_{t}, \Gamma_{t}, b_{t}$ and $\bar{b}_{t}$ are specified in a contract, called a credit support annex (CSA), meant to mitigate counterparty risk.

Remark 5.1 The above presentation reflects a pre-default reduced-form modeling approach, under the immersion hypothesis between the reference filtration $\mathbb{F}$ and a full model 
filtration $\mathbb{G}$ given as $\mathbb{F}$ progressively enlarged by the default times of the parties. From the financial point of view, the immersion hypothesis is a "moderate dependence" assumption between the counterparty risk and the underlying exposure, which can be accepted in the case of counterparty risk on interest rate derivatives. See Crépey and Song (2014) for further developments in this regard. Note that we write $\widetilde{\mathbb{F}}$ and $\mathbb{F}$ there instead of, respectively, $\mathbb{F}$ and $\mathbb{G}$ here. Since, in the present paper, we only work with pre-default values, we likewise denote here by $\mathbb{E}_{t}, \Theta_{t}$ and $g_{t}$ what is denoted by $\widetilde{\mathbb{E}}_{t}, \widetilde{\Theta}_{t}$ and $\widetilde{g}_{t}$ there.

In the numerical implementation which follows we set the above parameters equal to the following constants:

$$
\begin{aligned}
& \gamma^{b}=5 \%, \gamma^{c}=7 \%, \gamma=10 \% \\
& \rho^{b}=\rho^{c}=40 \% \\
& b=\bar{b}=\lambda=\widetilde{\lambda}=1.5 \%
\end{aligned}
$$

In view of the Markovian perspective of Sect. 3.5, we have $g_{t}(\vartheta)=\widehat{g}\left(t, X_{t}, \vartheta\right)$, for a suitable deterministic function $\widehat{g}$. Thus, (55) is rewritten as the following TVA Markovian BSDE:

$$
\Theta\left(t, X_{t}\right)=\mathbb{E}_{t}\left(\int_{t}^{\bar{T}} \widehat{g}\left(s, X_{s}, \Theta\left(s, X_{s}\right)\right) d s\right), t \in[0, \bar{T}] .
$$

Due to the specific choice of numerical parameters $\lambda=\widetilde{\lambda}$ above, the coefficients of the $\left(P_{t}-\vartheta-\Gamma_{t}\right)^{ \pm}$terms coincide in (57), so that this is the case of a "linear TVA" where the coefficient $g$ depends linearly on $\vartheta$. This will allow us to validate the results of the numerical BSDE scheme (61) for 60 by a standard Monte Carlo procedure.

\subsection{Basis Swap}

We illustrate numerically the above methodology on a basis swap with notional $N=100$ and maturity $\bar{T}=10 \mathrm{y}$, in the calibrated model of Sect. 4. The formula (21) yields the time0 basis swap spread $K_{0}^{b s w}=15 \mathrm{bps}$, which is added to the $3 \mathrm{~m}$ leg so that the basis swap is incepted at par. The first step ("forwardation") is to simulate, forward in time by an Euler scheme, a stochastic grid with $n$ (fixed to 100 below) uniform time steps and $m$ (set equal to $10^{4}$ or $10^{5}$ ) scenarios for the processes $r_{t}$ and $q_{t}$ and for the corresponding values $P_{t}$ of the basis swap, based on $P_{t}=P\left(t, X_{t}\right)$, with $X_{t}=\left(r_{t}, q_{t}, r_{t}^{1}, q_{t}^{1}, r_{t}^{2}, q_{t}^{2}\right)$ (cf. (46)). We use the standard algorithm in Glasserman (2003) to simulate the compound Poisson component of $Y^{2}$ and the method of Janicki and Weron (1993) for simulation of the FMLS component. The simulation of the rates $F_{t}(T, S)$ can be done without any discretization error since $a^{*}=0$. The results are plotted in Figure 5. Note that $q_{t}$, showed here for completeness, is a fudge factor without immediate financial meaning, so that the "large" values of $q_{t}$ compared with $r_{t}$ are not a real issue. The second step is to compute the TVA process, backward in time, by nonlinear regression on the time-space grid generated in the first step. We thus approximate $\Theta_{t}(\omega)$ in $(60)$ by $\widehat{\Theta}_{i}^{j}$ on the corresponding time-space grid, where the time-index $i$ runs from 1 to $n$ and the space-index $j$ runs from 1 to $m$. Denoting by $\widehat{\Theta}_{i}=\left(\widehat{\Theta}_{i}^{j}\right)_{1 \leq j \leq m}$ the vector of TVA values on the space grid at time $i$, we have $\widehat{\Theta}_{n}=0$ and then, for every $i=n-1, \cdots, 0$ and $j=1, \cdots, m$

$$
\widehat{\Theta}_{i}^{j}=\widehat{\mathbb{E}}_{i}^{j}\left(\widehat{\Theta}_{i+1}+\widehat{g}_{i+1}\left(\widehat{X}_{i+1}, \widehat{\Theta}_{i+1}\right) h\right),
$$



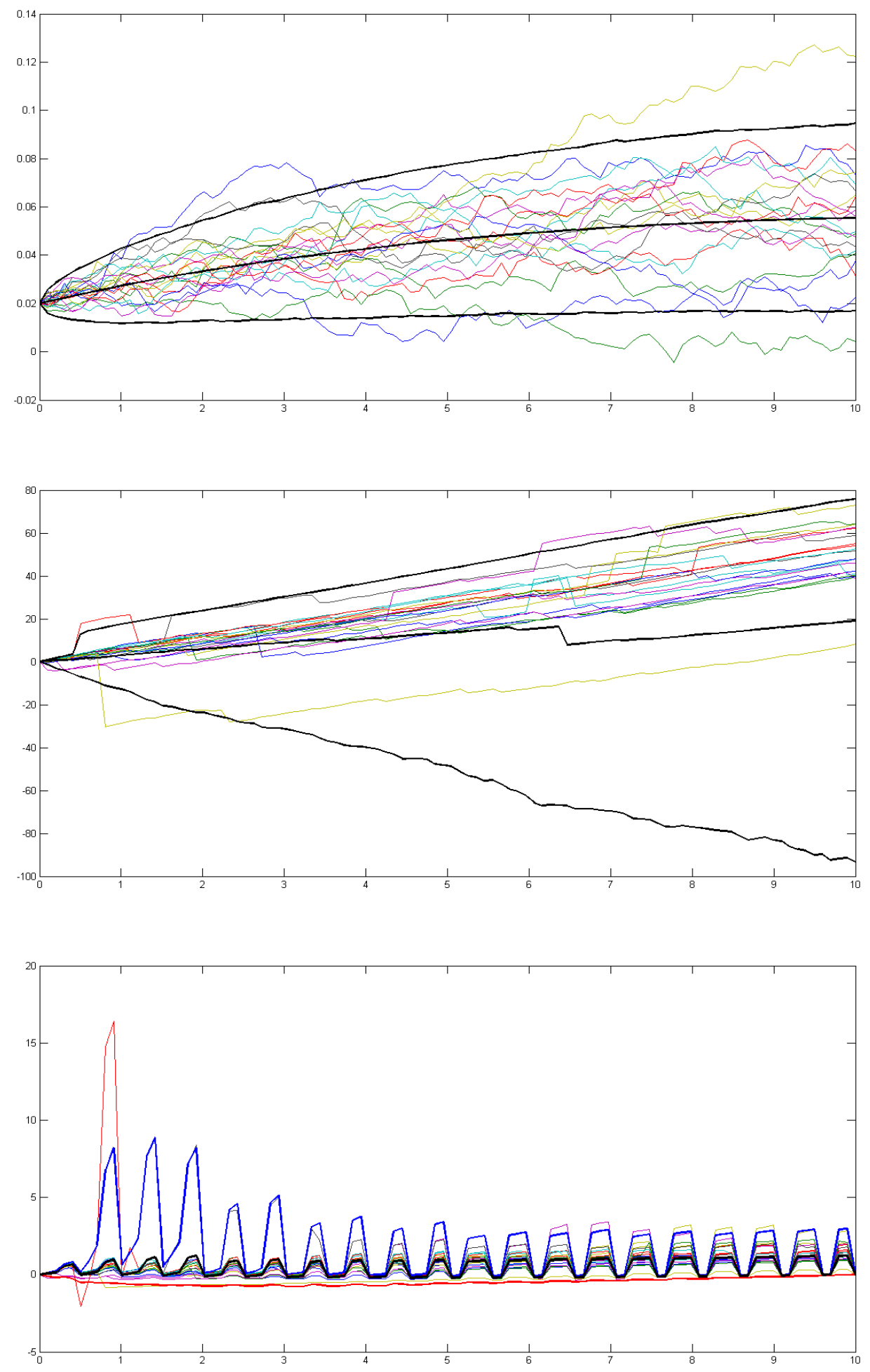

Figure 5: Clean valuation of the basis swap. Top panels: Processes $r_{t}$ and $q_{t}$. Bottom panel: Clean price process $P_{t}=P\left(t, X_{t}\right)$ of the basis swap where $X_{t}=\left(r_{t}, q_{t}, r_{t}^{1}, q_{t}^{1}, r_{t}^{2}, q_{t}^{2}\right)$. Each panel shows twenty paths simulated with $n=100$ time points, along with the process mean and $2.5 / 97.5$ percentiles computed as a function of time over $m=10^{4}$ simulated paths. 
for the time-step $h=\frac{\bar{T}}{n}=0.1 \mathrm{y}$. Here $\widehat{\mathbb{E}}_{i}^{j}$ is the conditional expectation given $\widehat{X}_{i}=\widehat{X}_{i}^{j}$, where $\widehat{X}_{i}^{j}$ is the $j^{\text {th }}$ simulated value of $X$ at the time step $i$. We use a $d$ nearest neighbor average non-parametric regression estimate (see e.g. Hastie, Tibshirani, and Friedman $(2009))$ with $d=1$, which was found the most efficient. This means that a conditional expectation of the form $\mathbb{E}(Y \mid X=x)$ is estimated by an empirical average of the three realizations of $Y$ associated with the three realizations of $X$ closest to $x$ in the Euclidean norm. In view of (45) and (46), this regression should in principle be performed against the "full" factor process $X_{t}=\left(r_{t}, q_{t}, r_{t}^{1}, q_{t}^{1}, r_{t}^{2}, q_{t}^{2}\right)$, where the last four components reflect the path-dependence of payments in arrears. However, as already noted in the single-curve setup of Crépey et al. (2013), these extra factors have a limited impact in practice. To illustrate this, Figure 6 shows the TVA processes obtained by regression for $m=10^{4}$ (top) and $10^{5}$ (bottom) against $r_{t}$ and $q_{t}$ only (left) and against the whole vector $X_{t}$ (right). Note that one should really target $m=10^{4}$ because in the industry practice one typically cannot afford much more on such applications, where not a single product, but the whole OTC derivative book of the bank, has to be dealt with. Table 1 displays the time- 0 value of the TVA and its CVA, DVA, LVA and RC components, where the components are obtained by substituting for $\vartheta$, in the respective term of (57), the TVA process $\Theta_{t}$ computed in the first place (see Subsect. 5.2 in Crépey et al. (2013) for the details of this procedure). The sum of the CVA, DVA, LVA and RC, which in theory equals the TVA, is shown in column 8. Therefore, columns 3,8 and 9 yield three different estimates for $\Theta_{0}$. Table 2 displays the relative differences between these estimates, as well as the Monte Carlo confidence interval in a comparable scale in the last column. As will also be the case with the basis swaption below (see Table 5), the TVA repriced by the sum of its components seems to be more accurate than the regressed TVA, an observation consistent with the better performance of Longstaff and Schwartz as compared with Tsitsiklis and Van Roy in American Monte Carlo methods (see e.g. Chapter 10 in Crépey (2013)). The expected exposure profiles (see Crépey et al. (2013) for the details about such representations) corresponding to the TVA decompositions of columns 4 to 7 in Table 1 are shown in Figure 7 for the regression against $(r, q)$ with $m=10^{4}$ paths. We only show the results in this case since the profiles in all the other three cases of Table 1 were found to be visually indistinguishable from the former.

\begin{tabular}{|r||r|r|r|r|r|r|r||r|}
\hline $\mathrm{m}$ & Regr & Regr TVA & CVA & DVA & LVA & RC & Sum & MC TVA \\
\hline \multirow{2}{*}{$10^{4}$} & r,q & -0.1224 & -0.1777 & 0.0191 & -0.0422 & 0.0780 & -0.1227 & -0.1217 \\
\cline { 2 - 9 } & PD & -0.1246 & -0.1777 & 0.0191 & -0.0420 & 0.0790 & -0.1216 & \\
\hline \multirow{2}{*}{$10^{5}$} & r,q & -0.1256 & -0.1817 & 0.0205 & -0.0427 & 0.0792 & -0.1246 & \multirow{2}{*}{-0.1239} \\
\cline { 2 - 9 } & PD & -0.1252 & -0.1817 & 0.0205 & -0.0427 & 0.0793 & -0.1246 & \\
\hline
\end{tabular}

Table 1: Time-0 TVA and its decomposition (all in $€$ ) computed by regression for $m=10^{4}$ or $10^{5}$ against $r_{t}$ and $q_{t}$ only (rows "r,q") or against the whole vector $X_{t}$ accounting for path-dependence due to the payments in arrears (rows "PD"). Column 3: Time-0 regressed TVA. Columns 4 to $\%$ : TVA decomposition into time-0 CVA, DVA, LVA and RC repriced individually by plugging $\Theta_{t}$ for $\vartheta$ in the respective term of (57). Column 8: Sum of the four components. Column 9: Monte Carlo TVA.

Next, to compare alternative CSA specifications, we repeat the above numerical implementation in each of the following five cases, with $\bar{\lambda}$ set equal to $4.5 \%$ everywhere (and 

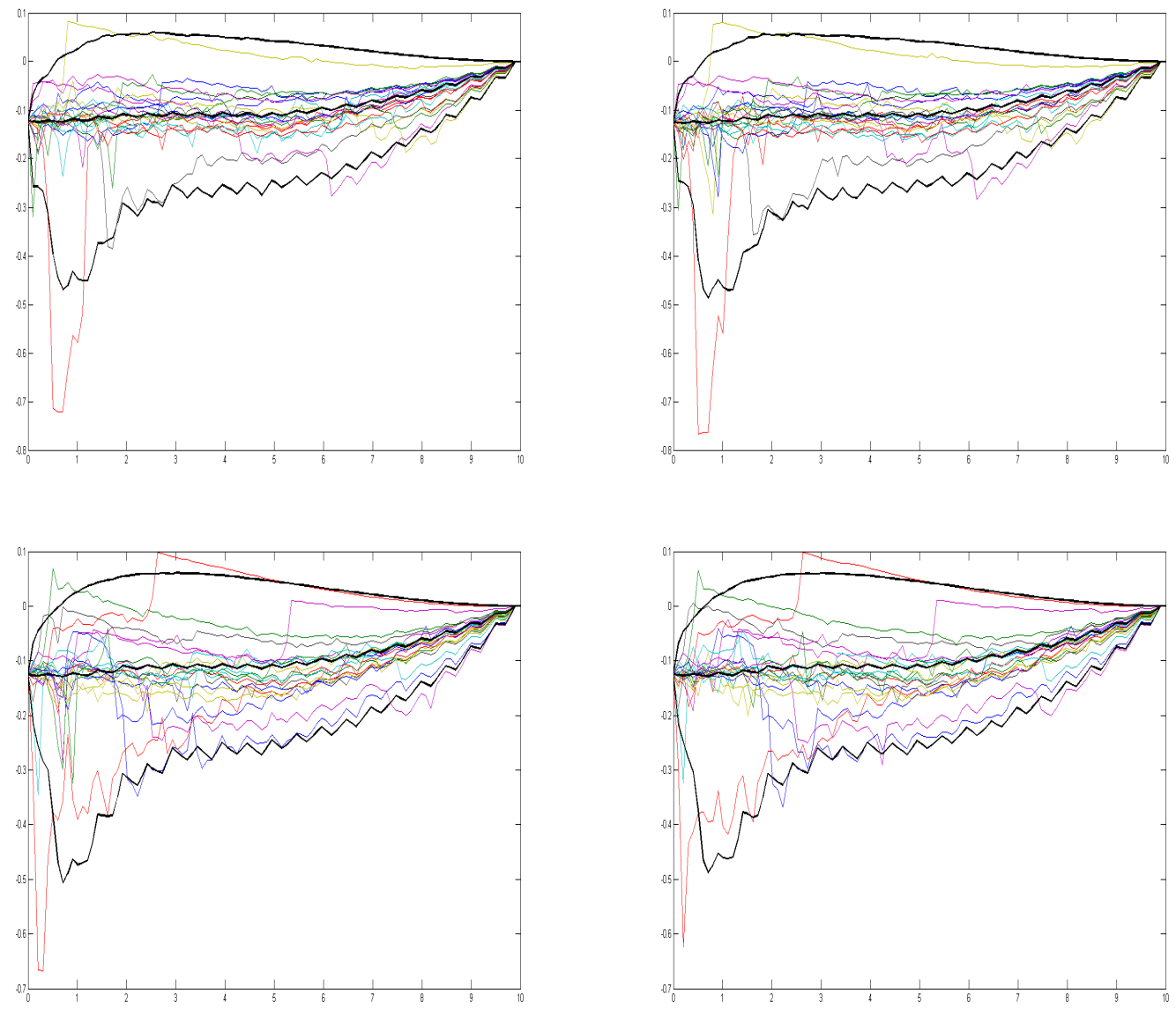

Figure 6: TVA process $\Theta_{t}$ of the basis swap. All graphs show twenty paths of the TVA process at $n=100$ time points, along with the process mean and $2.5 / 97.5$ percentiles as a function of time. Top graphs: $m=10^{4}$ simulated paths. Bottom graphs: $m=10^{5}$ simulated paths. Left graphs: "Reduced" regression against $\left(r_{t}, q_{t}\right)$. Right graphs: "Full" regression against $X_{t}=\left(r_{t}, q_{t}, r_{t}^{1}, q_{t}^{1}, r_{t}^{2}, q_{t}^{2}\right)$.

\begin{tabular}{|r||r|r|r|r|r|}
\hline $\mathrm{m}$ & Regr & Sum/TVA & TVA/MC & Sum/MC & CI//|MC| \\
\hline \multirow{2}{*}{$10^{4}$} & $\mathrm{r}, \mathrm{q}$ & $0.25 \%$ & $0.58 \%$ & $0.82 \%$ & \multirow{2}{*}{$2.88 \%$} \\
\cline { 2 - 5 } & $\mathrm{PD}$ & $-2.41 \%$ & $2.38 \%$ & $-0.08 \%$ & \\
\hline \multirow{2}{*}{$10^{5}$} & $\mathrm{r}, \mathrm{q}$ & $-0.80 \%$ & $1.37 \%$ & $0.56 \%$ & \multirow{2}{*}{$1.25 \%$} \\
\cline { 2 - 5 } & $\mathrm{PD}$ & $-0.48 \%$ & $1.05 \%$ & $0.56 \%$ & \\
\hline
\end{tabular}

Table 2: Time-0 TVA relative errors corresponding to the results of Table 1. "A/B" represents the relative difference $\frac{(A-B)}{B}$. "CI//|MC|" in the last column refers to the half-size of the $95 \%$-Monte Carlo confidence interval divided by the absolute value of the standard Monte Carlo estimate of the time-0 TVA. 

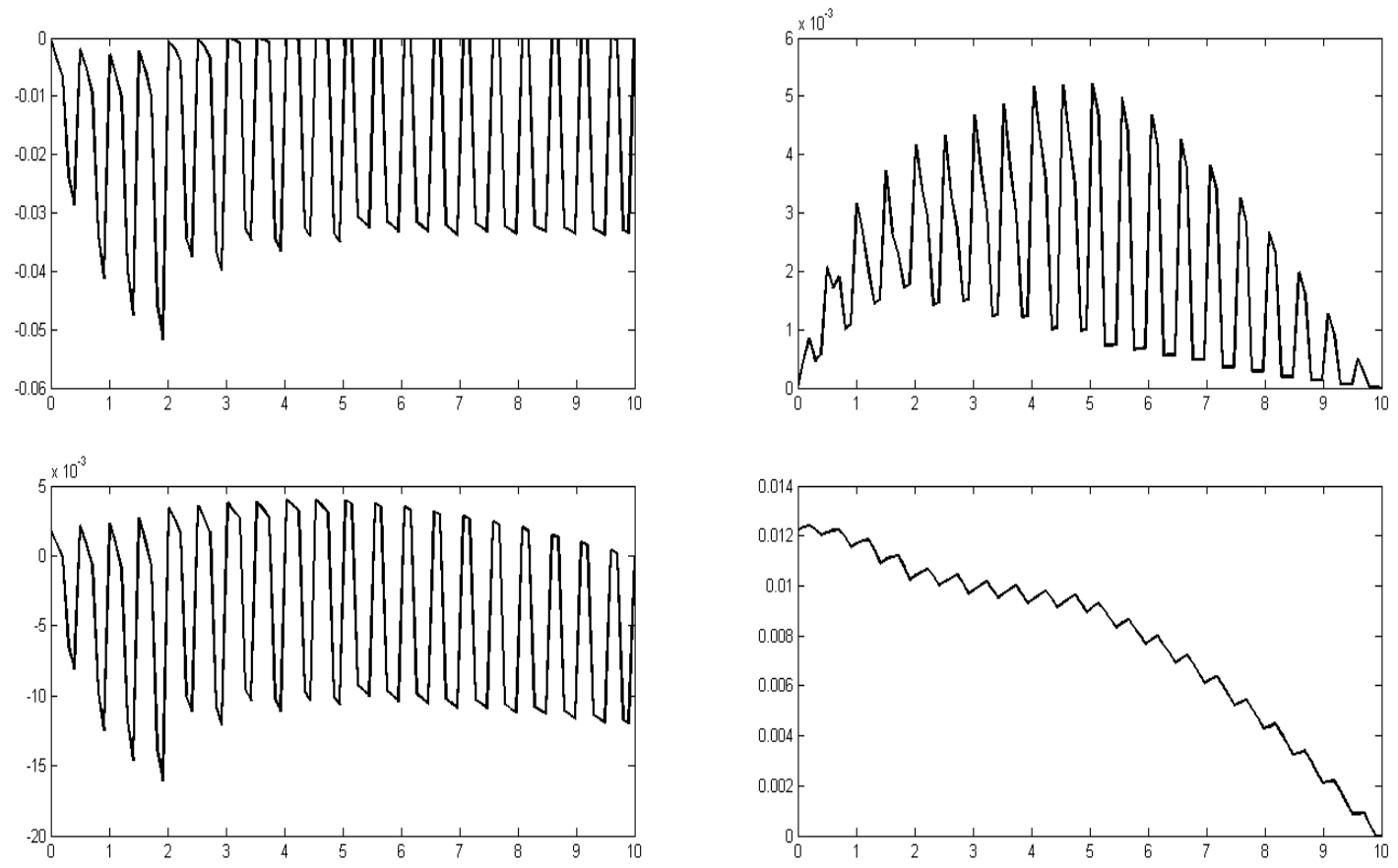

Figure 7: Expected exposures of the TVA components of the basis swap. Top panels: CVA (left) and DVA (right) exposures. Bottom panels: LVA (left) and RC (right) exposures.

the other parameters as in $(59))$ :

1. $\left(\mathfrak{r}^{b}, \rho^{b}, \rho^{c}\right)=(100,40,40) \%, \quad Q=P, \quad \Gamma=0$

$2 \quad\left(\mathfrak{r}^{b}, \rho^{b}, \rho^{c}\right)=(100,40,40) \%, \quad Q=P, \quad \Gamma=Q=P$

3. $\quad\left(\mathfrak{r}^{b}, \rho^{b}, \rho^{c}\right)=(40,40,40) \%, \quad Q=P, \quad \Gamma=0$

4. $\quad\left(\mathfrak{r}^{b}, \rho^{b}, \rho^{c}\right)=(100,100,40) \%, \quad Q=P, \quad \Gamma=0$

5. $\quad\left(\mathfrak{r}^{b}, \rho^{b}, \rho^{c}\right)=(100,100,40) \%, \quad Q=\Pi, \quad \Gamma=0$

(same cases as in Crépey, Gerboud, Grbac, and Ngor (2013), for comparison purposes). Note that in case 3, we have by (58):

$$
\widetilde{\lambda}=4.5 \%-0.6 \times 0.5 \times 10 \%=1.5 \%=\lambda,
$$

so this is the linear TVA case considered above. Table 3 shows an analog of the first row of Table 1 (time-0 TVA and its decomposition for $m=10^{4}$ and regression against $(r, q)$ in each of the five cases). Moving from case 1 to 2, there are no CVA and DVA anymore and the dominant effect is the cancelation of the previously highly negative, costly CVA, resulting in a higher TVA, hence a lower (selling) price for the bank. Moving from 1 to 3 , a funding benefit at own default is acknowledged by the bank, resulting in a higher TVA, hence a lower selling price for the bank. Moving from 1 to 4, the beneficial DVA at own default is ignored by the bank, being considered as fake benefit, which results in a lower TVA, but negligibly so, since the DVA was very small anyway. The related LVA numbers are very close because the parameter $\rho^{b}$, which changes between these two cases, has no direct impact on the LVA and the indirect impact through the change of the TVA in the second row of (57) is limited (as the parameters $\lambda$ and $\tilde{\lambda}$ are not so large). Finally, 5 represents a 
(slightly artificial) case of a bank in a "dominant" position, able to enforce the value of the contract $\Pi$ from its own perspective (see (56)) for the CSA close-out valuation process $Q$. Hence, a zero RC for the bank follows in this case.

\begin{tabular}{|r||r|r|r|r|r|r|r|}
\hline Case & Regr TVA & CVA & DVA & LVA & RC & Sum & Sum/TVA \\
\hline 1 & -0.1823 & -0.1777 & 0.0191 & -0.1427 & 0.1183 & -0.1829 & $0.33 \%$ \\
\hline 2 & -0.0311 & 0 & 0 & -0.0510 & 0.0200 & -0.0311 & $0.00 \%$ \\
\hline 3 & -0.1224 & -0.1777 & 0.0191 & -0.0422 & 0.0780 & -0.1227 & $0.24 \%$ \\
\hline 4 & -0.1939 & -0.1777 & 0 & -0.1412 & 0.1241 & -0.1948 & $0.46 \%$ \\
\hline 5 & -0.2743 & -0.1427 & 0 & -0.1324 & 0 & -0.2751 & $0.29 \%$ \\
\hline
\end{tabular}

Table 3: Time-0 TVA and its decomposition (all in $€$ ) computed by regression for $m=$ $10^{4}$ against $r_{t}$ and $q_{t}$. Column 2: Time-0 regressed TVA $\Theta_{0}$. Columns 3 to 6 : TVA decomposition into time-0 CVA, DVA, LVA and RC repriced individually by plugging $\Theta_{t}$ for $\vartheta$ in the respective term of (57). Column \%: Sum of the four components. Column 8: Relative difference between columns 7 and 2 .

\subsection{Basis Swaption}

Figures 8,10 and Tables 46 are the analogs of Figures 5, 5 and Tables 1,3 for a swaption with maturity 5 years on the above basis swap, with the remaining lifetime of 5 years at that time point (swaption with payoff $\left(P_{5 y}^{b s w}\right)^{+}$, where $P_{5 y}^{b s w}$ is given by 20 with $K=K_{0}^{b s w}$ and $t=5 y)$. Note that no explicit formulas for $P_{t}$ were used in these computations. Instead, $P_{t}$ is computed by simulation/regression on the same stochastic grid as the TVA, backward starting from $t=5 y=t_{\nu}$, where $\nu=\frac{n}{2}$, based on the following dynamic programming recursion (compare with (61)): $\widehat{P}_{\nu}=\left(P_{5 y}^{b s w}\right)^{+}$at grid points at time $t_{\nu}$, and then for every $i=\nu-1, \cdots, 0$ and $j=1, \cdots, m$,

$$
\widehat{P}_{i}^{j}=\widehat{\mathbb{E}}_{i}^{j}\left(\left(1-\widehat{r}_{i+1} h\right) \widehat{P}_{i+1}\right),
$$

where $\widehat{r}_{i+1}$ is the simulated value of $r$ at the time step $(i+1)$. The time- 0 price of the basis swaption is $P_{0}=1.02 €$ (by Monte Carlo with $m=10^{5}$ paths) and the time-0 TVA is quite large (depending on the CSA specification) compared with this. As visible in Table 5 , the nonlinear regression TVA estimates (time-0 TVA computed by regression or repriced as the sum of its four components) are mainly outside the corresponding Monte Carlo confidence intervals, especially for $m=10^{5}$, but this is not surprising since these estimates entail space-regression biases when compared with a standard Monte Carlo estimate (but of course the latter is no longer available in nonlinear cases). Observe from Table 6 that since the basis swaption is always in-the-money for the bank, there is no DVA in this case, thus CSA specifications 1 and 4 are equivalent here.

\section{Acknowledgements}

We thank Giovanni Cesari and Andrea Pallavicini for their comments on preliminary versions of this paper, as well as Rama Cont and other participants of the Conference on Current Topics in Mathematical Finance (WU Vienna, April 2013), where this work was 


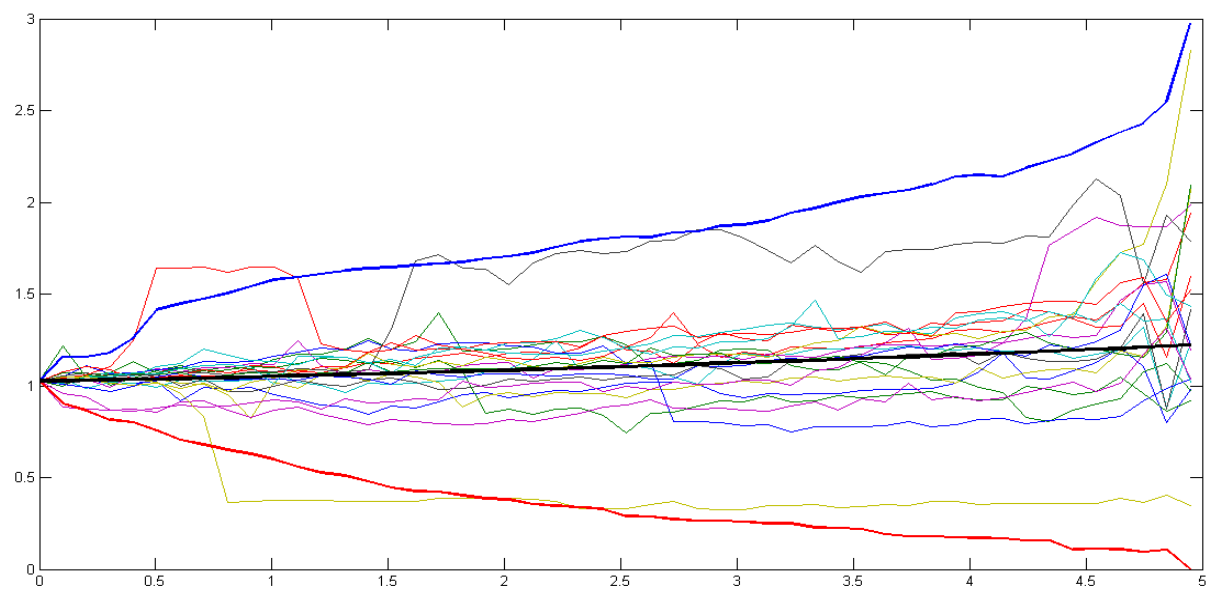

Figure 8: Clean price process $P_{t}=P\left(t, X_{t}\right)$ of the basis swaption computed by regression on the simulated factors grid.
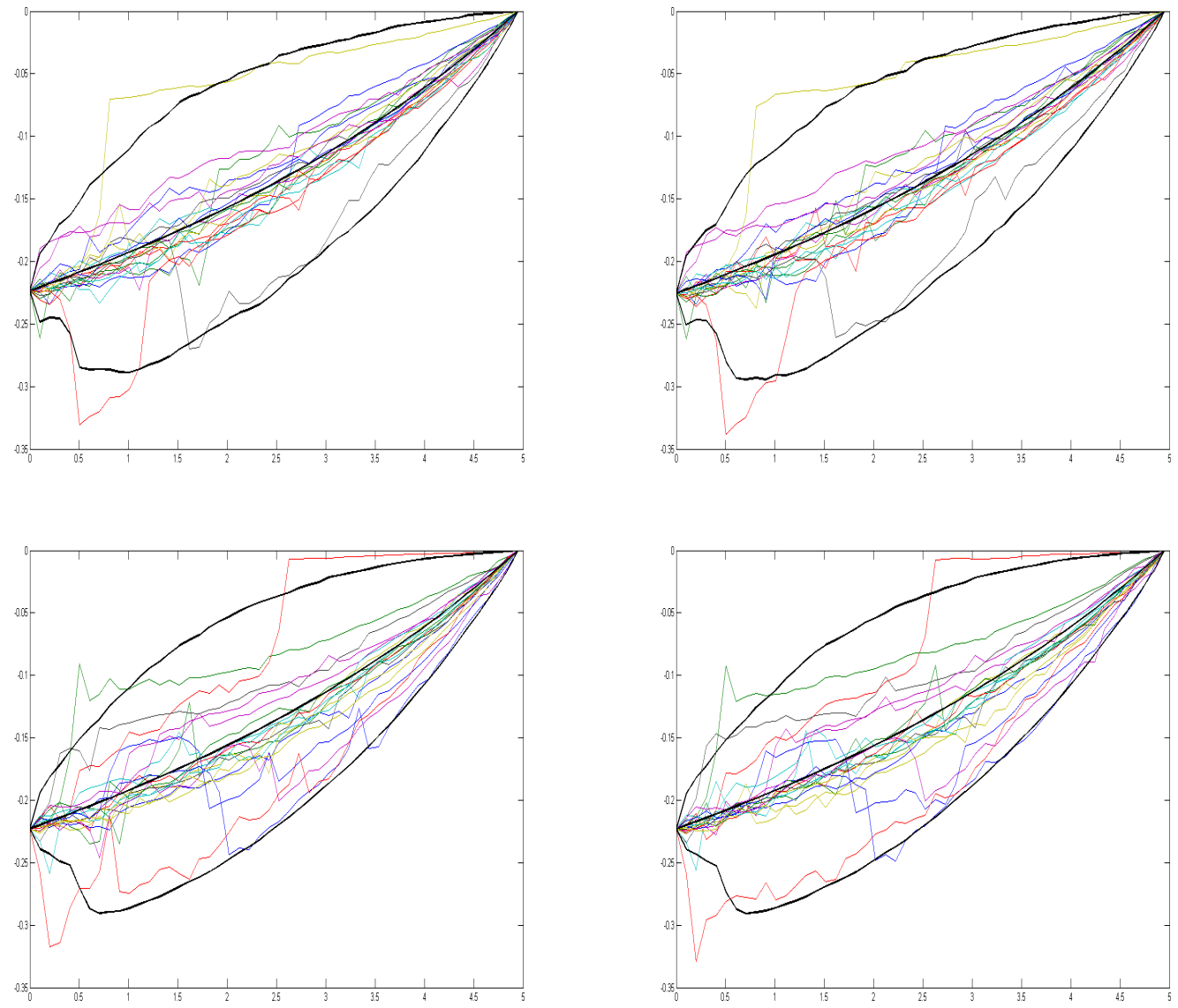

Figure 9: TVA process $\Theta_{t}$ of the basis swaption. 


\begin{tabular}{|r||r|r|r|r|r|r|r|r|}
\hline $\mathrm{m}$ & Regr & Regr TVA & CVA & DVA & LVA & RC & Sum & MC TVA \\
\hline \multirow{2}{*}{$10^{4}$} & r,q & -0.2235 & -0.2133 & 0 & -0.0672 & 0.0596 & -0.2209 & -0.2194 \\
\cline { 2 - 9 } & PD & -0.2255 & -0.2144 & 0 & -0.0676 & 0.0600 & -0.2220 & -0.2207 \\
\hline \multirow{2}{*}{$10^{5}$} & r,q & -0.2228 & -0.2124 & 0 & -0.0669 & 0.0593 & -0.2200 & -0.2185 \\
\cline { 2 - 9 } & PD & -0.2230 & -0.2125 & 0 & -0.0670 & 0.0594 & -0.2201 & -0.2187 \\
\hline
\end{tabular}

Table 4: Basis swaption: Time-0 TVA and its decomposition.

\begin{tabular}{|r||r|r|r|r||r|}
\hline $\mathrm{m}$ & Regr & Sum/TVA & TVA/MC & Sum/MC & CI//|MC| \\
\hline \multirow{2}{*}{$10^{4}$} & r,q & $-1.16 \%$ & $1.87 \%$ & $0.68 \%$ & $0.43 \%$ \\
\cline { 2 - 6 } & PD & $-1.55 \%$ & $2.17 \%$ & $0.59 \%$ & $0.43 \%$ \\
\hline \multirow{2}{*}{$10^{5}$} & r,q & $-1.26 \%$ & $1.97 \%$ & $0.69 \%$ & $0.14 \%$ \\
\cline { 2 - 6 } & PD & $-1.30 \%$ & $1.97 \%$ & $0.64 \%$ & $0.16 \%$ \\
\hline
\end{tabular}

Table 5: Basis swaption: Time-0 TVA relative errors.
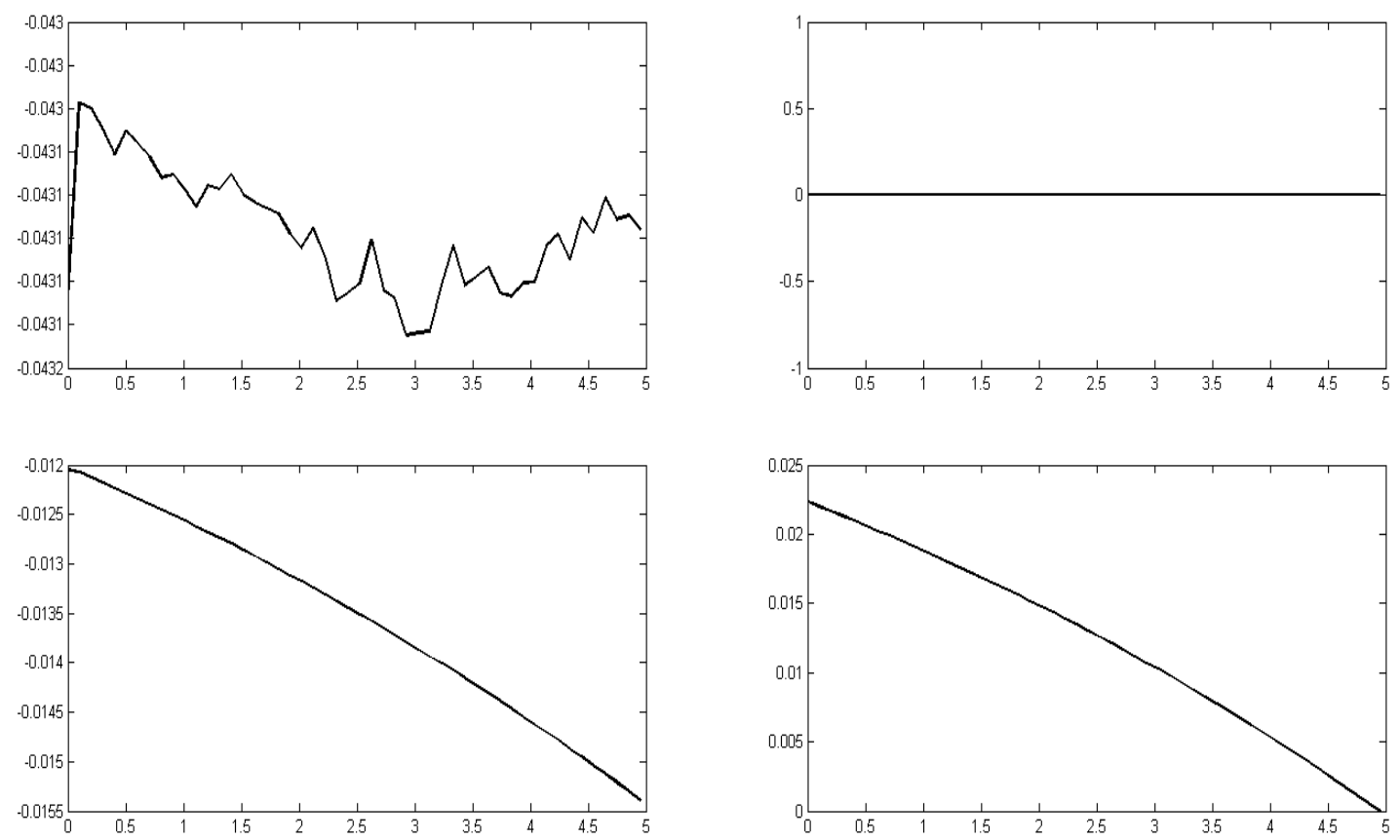

Figure 10: Expected exposures of the TVA components of the basis swaption.

\begin{tabular}{|r||r|r|r|r|r|r|r|}
\hline Case & Regr TVA & CVA & DVA & LVA & RC & Sum & Sum/TVA \\
\hline 1 & -0.3192 & -0.2133 & 0 & -0.1893 & 0.0871 & -0.3156 & $-1.13 \%$ \\
\hline 2 & -0.0588 & 0 & 0 & -0.0738 & 0.0157 & -0.0581 & $-1.19 \%$ \\
\hline 3 & -0.2235 & -0.2133 & 0 & -0.0672 & 0.0596 & -0.2209 & $-1.16 \%$ \\
\hline 4 & -0.3192 & -0.2133 & 0 & -0.1893 & 0.0871 & -0.3156 & $-1.13 \%$ \\
\hline 5 & -0.3635 & -0.1735 & 0 & -0.1859 & 0 & -0.3593 & $-1.16 \%$ \\
\hline
\end{tabular}

Table 6: Basis swaption: Time-0 TVA and its decomposition under the 5 CSA specifications 
first presented, for useful questions and remarks. We would like to thank two anonymous referees for their valuable comments.

\section{References}

Akkara, G. (2012). OIS discounting and dual-curve stripping methodology at Bloomberg. Bloomberg Documentation.

Bianchetti, M. (2010). Two curves, one price. Risk Magazine, August 74-80.

Bianchetti, M. and M. Morini (Eds.) (2013). Interest Rate Modelling After the Financial Crisis. Risk Books.

Brigo, D., M. Morini, and A. Pallavicini (2013). Counterparty Credit Risk, Collateral and Funding: With Pricing Cases For All Asset Classes. Wiley.

Carr, P. and L. Wu (2003). The finite moment log stable process and option pricing. The Journal of Finance 58(2), 753-778.

Cont, R. and P. Tankov (2003). Financial Modelling with Jump Processes. Chapman and Hall/CRC Press.

Crépey, S. (2012). Bilateral counterparty risk under funding constraints - Part II: CVA. Mathematical Finance. Forthcoming, DOI 10.1111/mafi.12005.

Crépey, S. (2013). Financial Modeling: A Backward Stochastic Differential Equations Perspective. Springer Finance Textbooks.

Crépey, S., T. R. Bielecki, and D. Brigo (2014). Counterparty Risk and Funding-A Tale of Two Puzzles. Chapman \& Hall/CRC Financial Mathematics Series. June 2014.

Crépey, S., R. Gerboud, Z. Grbac, and N. Ngor (2013). Counterparty risk and funding: The four wings of the TVA. International Journal of Theoretical and Applied Finance 16(2), 1350006 (31 pages).

Crépey, S., Z. Grbac, and H. N. Nguyen (2012). A multiple-curve HJM model of interbank risk. Mathematics and Financial Economics 6 (3), 155-190.

Crépey, S. and S. Song (2014). Counterparty risk modeling: Beyond immersion. Submitted.

Duffie, D., J. Pan, and K. Singleton (2000). Transform analysis and asset pricing for affine jump-diffusions. Econometrica 68(6), 1343-1376.

Eberlein, E., K. Glau, and A. Papapantoleon (2010). Analysis of Fourier transform valuation formulas and applications. Applied Mathematical Finance 17(3), 211-240.

Eberlein, E. and F. Özkan (2005). The Lévy Libor model. Finance and Stochastics 9, $327-348$.

Eberlein, E. and S. Raible (1999). Term structure models driven by general Lévy processes. Mathematical Finance 9, 31-53.

Filipović, D. and A. B. Trolle (2013). The term structure of interbank risk. Journal of Financial Economics $109(3), 707-733$.

Fujii, M., Y. Shimada, and A. Takahashi (2010). A note on construction of multiple swap curves with and without collateral. FSA Research Review 6, 139-157. 
Fujii, M., Y. Shimada, and A. Takahashi (2011). A market model of interest rates with dynamic basis spreads in the presence of collateral and multiple currencies. Wilmott Magazine 54, 61-73.

Fujii, M. and A. Takahashi (2011). Choice of collateral currency. Risk Magazine, January $120-125$.

Glasserman, P. (2003). Monte Carlo Methods in Financial Engineering. Springer-Verlag.

Hagan, P. S. and G. West (2006). Interpolation methods for curve construction. Applied Mathematical Finance 13(2), 89-129.

Hastie, T., R. Tibshirani, and J. Friedman (2009). The Elements of Statistical Learning: Data Mining, Inference, and Prediction. Springer.

Henrard, M. (2007). The irony in the derivatives discounting. Wilmott Magazine 30, 92-98.

Henrard, M. (2010). The irony in the derivatives discounting part II: the crisis. Wilmott Magazine 2, 301-316.

Hubalek, F. and J. Kallsen (2005). Variance-optimal hedging and Markowitz-efficient portfolios for multivariate processes with stationary independent increments with and without constraints. Working paper, TU München.

Hurd, T. R. and Z. Zhou (2010). A Fourier transform method for spread option pricing. SIAM Journal on Financial Mathematics 1, 142-157.

Janicki, A. and A. Weron (1993). Simulation and Chaotic Behavior of Alpha-stable Stochastic Processes, Volume 178. CRC Press.

Kenyon, C. (2010). Short-rate pricing after the liquidity and credit shocks: including the basis. Risk Magazine, November 83-87.

Kienitz, J. (2013). Transforming volatility - multi curve cap and swaption volatilities. Available at SSRN: http://ssrn.com/abstract=2204702.

Kijima, M., K. Tanaka, and T. Wong (2009). A multi-quality model of interest rates. Quantitative Finance 9(2), 133-145.

Levin, K. and J. X. Zhang (2014). The Bloomberg Volatility Cube. Documentation Bloomberg L.P.

Mercurio, F. (2010a). A LIBOR market model with stochastic basis. Risk Magazine, December 84-89.

Mercurio, F. (2010b). Interest rates and the credit crunch: new formulas and market models. Technical report, Bloomberg Portfolio Research Paper No. 2010-01-FRONTIERS.

Moreni, N. and A. Pallavicini (2013). Parsimonious multi-curve HJM modelling with stochastic volatility. In M. Bianchetti and M. Morini (Eds.), Interest Rate Modelling After the Financial Crisis. Risk Books.

Moreni, N. and A. Pallavicini (2014). Parsimonious HJM modelling for multiple yieldcurve dynamics. Quantitative Finance 14(2), 199-210.

Pallavicini, A., D. Perini, and D. Brigo (2012). Funding, collateral and hedging: uncovering the mechanics and the subtleties of funding valuation adjustments. http://dx.doi.org/10.2139/ssrn.2161528. 
Sato, K.-I. (1999). Lévy Processes and Infinitely Divisible Distributions. Cambridge University Press.

Schrager, D. F. and A. A. Pelsser (2006). Pricing swaptions and coupon bond options in affine term structure models. Mathematical Finance 16(4), 673-694.

Singleton, K. and L. Umantsev (2002). Pricing coupon-bond options and swaptions in affine term structure models. Mathematical Finance 12, 427-446. 